\title{
Activated escape over oscillating barriers: The case of many dimensions
}

\author{
Jörg Lehmann, ${ }^{1}$ Peter Reimann, ${ }^{2}$ and Peter Hänggi ${ }^{1}$ \\ ${ }^{1}$ Institut für Physik, Universität Augsburg, Universitätsstraße 1, D-86135 Augsburg, Germany \\ ${ }^{2}$ Fakultät für Physik, Universität Bielefeld, Universitätsstraße 25, D-33615 Bielefeld, Germany
}

(Dated: October 29, 2018)

\begin{abstract}
We present a novel path-integral method for the determination of time-dependent and timeaveraged reaction rates in multidimensional, periodically driven escape problems at weak thermal noise. The so obtained general expressions are evaluated explicitly for the situation of a sinusoidally driven, damped particle with inertia moving in a metastable, piecewise parabolic potential. A comparison with data from Monte-Carlo simulations yields a very good agreement with our analytic results over a wide parameter range.
\end{abstract}

PACS numbers: 05.40.-a, 82.20.Mj, 82.20.Pm

\section{INTRODUCTION}

Thermally activated escape problems in the presence of an explicit time-dependent driving are at the root of many timely transport processes. Typical examples comprise the control of chemical reactions with tailored laser pulses [1, 2], ion transport through voltage-gated channels [3], the pumping and shuttling of particles in Brownian environments 4, 5, 6, 7, 8, 9], or the amplification of weak information-carrying signals via the phenomenon of Stochastic Resonance 110, 11, 12, 13, to name only a few. In the absence of such a time-dependent driving and for the case of weak thermal noise the escape time is governed - as commonly known - by an exponentially leading Arrhenius factor [14, 15, 16]. Pioneered by Kramers [17] and extended to arbitrary dimensions in the works [18, 19, 20, 21, this scheme, however, meets formidable difficulties under far from thermal equilibrium conditions. This is so because of an extremely complex interplay between the global properties of the metastable potential and the nonlinear noisy dynamics 14, 22, 23, 24. The subject of our present paper is one of the simplest and experimentally most natural such non-equilibrium descendants of Kramers' original escape problem [17], namely the thermally activated escape of a Brownian particle over a potential barrier in the presence of periodic driving which modulates both the corresponding potential well region and the activation barrier. While most previous attempts have been restricted to weak $25,26,27$, slow [28, 29], or fast [25, 28, 30] driving, we have addressed in recent analytical explorations 31, 32 by means of a path-integral technique the most challenging intermediate regime of moderately strong and moderately fast driving for a one-dimensional, overdamped escape problem. Closely related to our recent works are the subsequent appealing efforts in Ref. [33], wherein on uses instead the method of singular perturbation theory in the weak noise limit a so termed WKB approximation - which, however, also has been restricted to cover only the overdamped, onedimensional case. The scheme in Ref. [33 yields results which are consistent with the findings in Ref. 31, 32]; but it remains on a more formal and implicit level compared to ours.

The objective of this study is to extend these recent works of time-dependent rate theory put forward in Refs. 31, 32, 33 to the case of many dimension of the underlying stochastic process. In particular, we shall consider the generic case of a driven inertial Brownian motion dynamics. Furthermore, we shall derive for weak noise asymptotically exact results in analytically closed form for the escape of an inertial, sinusoidally driven Brownian particle in a metastable, piecewise parabolic potential in the regime of moderate forcing strengths and forcing frequencies. A comparison with data from Monte-Carlo simulations yields very good agreement over a wide parameter range.

We anticipate here that the following presentation is on purpose kept rather concise. While we are confident that the general idea of our approach remains more transparent in this way, it is nevertheless advisable to consult the detailed discussion of the one-dimensional, overdamped (i.e. no inertial) dynamics in Ref. [32] for a more thorough and in-depth understanding. It must be pointed out, however, that our present generalization to many dimensions in addition requires several conceptually new steps and features as compared to the one-dimensional overdamped case.

In Sect. II , we describe the model under investigation and define both the instantaneous and the time-averaged escape rate. The path-integral formalism and its evaluation for weak-noise is described in Sect. III. Its application to the escape problem is demonstrated in Sect. IV. Our general findings are then evaluated and discussed explicitly for the case of the driven Kramers problem with a piecewise parabolic potential in Sect. $\mathrm{V}$. Concluding remarks are given in Sect. VI. 


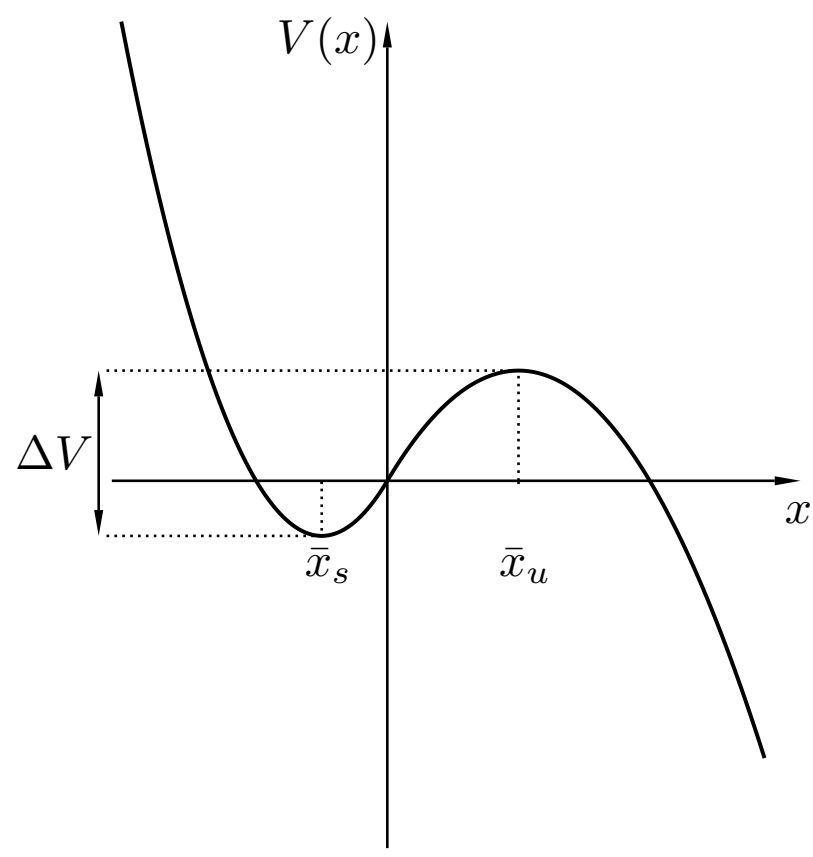

FIG. 1: Sketch of a typical metastable potential $V(x)$, namely the piecewise parabolic potential $(75)$ with $\Delta V=0.9, m \omega_{s}^{2}=0.6$, and $m \omega_{u}^{2}=-0.3$ in arbitrary, dimensionless units.

\section{THE ESCAPE PROBLEM}

\section{A. The general Model}

The starting-point of our investigations is the following model for the $d$-dimensional Brownian motion of a particle with coordinates $\mathbf{x}(t)$ in a time-dependent force field $\mathbf{F}(\mathbf{x}, t)$ :

$$
\dot{\mathbf{x}}(t)=\mathbf{F}(\mathbf{x}(t), t)+\sqrt{2 \epsilon} \mathrm{B} \boldsymbol{\xi}(t) .
$$

Here, bold quantities denote $d$-dimensional vectors, while $d \times d$-matrices are represented by sans-serif fonts. The $d$-dimensional Gaussian uncorrelated, white noise $\boldsymbol{\xi}(t)$ is defined by the relations $(i, j=1,2, \ldots, d)$

$$
\left\langle\xi_{i}(t)\right\rangle=0, \quad\left\langle\xi_{i}(t) \xi_{j}\left(t^{\prime}\right)\right\rangle=\delta_{i j} \delta\left(t-t^{\prime}\right),
$$

where $\delta_{i j}$ is the Kronecker delta and $\delta(t)$ Dirac's $\delta$-distribution. The additive coupling of the noise to the stochastic dynamics is determined by the matrix $\mathrm{B}$, which we assume to be non-singular.

We restrict ourselves to the case of a time-periodic driving, i.e. $\mathbf{F}(\mathbf{x}, t)=\mathbf{F}(\mathbf{x}, t+\mathcal{T})$ for a certain period $\mathcal{T}$. Furthermore, we assume that the deterministic dynamics, i.e. Eq. (1i) for $\epsilon=0$, possesses exactly one stable periodic orbit $\mathbf{x}_{s}(t)=\mathbf{x}_{s}(t+\mathcal{T})$ with a time-dependent domain of attraction $\mathcal{A}(t)$. All other deterministic orbits, which start outside of $\mathcal{A}(t)$ and its boundary $\mathcal{S}(t):=\partial \mathcal{A}(t)$ are assumed to diverge in the long time limit. The boundary $\mathcal{S}(t)$ then acts as a separatrix between these two kinds of deterministic solutions. Moreover, we require that there is exactly one unstable periodic orbit $\mathbf{x}_{u}(t)=\mathbf{x}_{u}(t+\mathcal{T})$, which then moves with the separatrix, i.e. $\mathbf{x}_{u}(t) \in S(t)$ for all times $t$. Finally, all other deterministic solutions starting in a neighborhood of the unstable periodic orbit on the separatrix are assumed to be bounded. All these assumptions can usually be taken for granted in typical periodically driven escape problems of practical interest.

A particularly prominent example is the Kramers problem [17], namely the escape of a particle with mass $m$ out of the bottom well of a static potential as cartooned in Fig. 11. Under the additional influence of an additive sinusoidal driving this corresponds to the stochastic dynamics

$$
m \ddot{x}+\eta \dot{x}=-V^{\prime}(x)+A \sin (\Omega t)+\sqrt{2 \eta k_{B} T} \xi(t) .
$$


Here, $\eta$ is the viscous friction coefficient, $k_{B}$ Boltzmann's constant, $T$ the temperature and $\Omega=2 \pi / \mathcal{T}$ the angular frequency of the driving. We can rewrite the second order equation (3) in the form of Eq. (11), if we identify

$$
\mathbf{x}:=\left(\begin{array}{l}
x \\
v
\end{array}\right), \quad \mathbf{F}(\mathbf{x}, t):=\left(\begin{array}{c}
v \\
\frac{1}{m} F(x, t)-\gamma v
\end{array}\right), \quad \mathrm{B}:=\frac{\sqrt{\eta}}{m}\left(\begin{array}{ll}
\delta & 0 \\
0 & 1
\end{array}\right), \quad \epsilon:=k_{B} T,
$$

where we have introduced the one-dimensional force field $F(x, t):=-V^{\prime}(x)+A \sin (\Omega t)$ and the frequency $\gamma:=\eta / m$. Furthermore, we have added an auxiliary noise source of a strength proportional to $\delta$ to ensure that B is non-singular. Eventually, we shall consider the limit $\delta \rightarrow 0$, in which Eq. (4) reduces to the original dynamics (3). Figure 2 depicts the stable and unstable periodic orbits, the domain of attraction $\mathcal{A}(t)$, and the separatrix $\mathcal{S}(t)$ for a representative metastable potential.

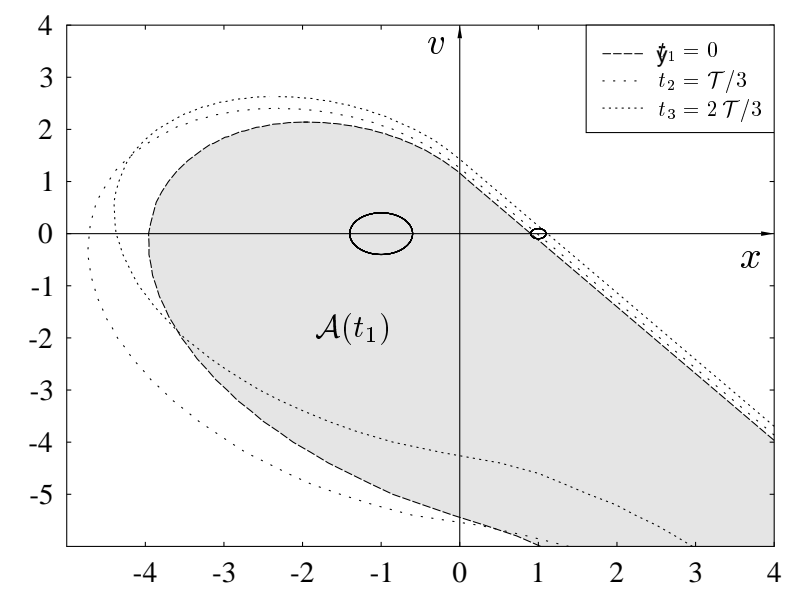

FIG. 2: Phase space diagram for a typical metastable potential with additive sinusoidal driving (3), namely the piecewise parabolic potential (75) with $\bar{x}_{s}=\omega_{u}^{2}=-1, \bar{x}_{u}=\omega_{s}^{2}=m=\Delta V=\Omega=1, \eta=0.5, A=0.2$. Solid line: Stable (left) and unstable (right) periodic orbit. Dashed lines: Separatrix at different times $t_{1}=0, t_{2}=\mathcal{T} / 3$, and $t_{3}=2 \mathcal{T} / 3$. Grey area: Domain of attraction $\mathcal{A}\left(t_{1}\right)$ of stable periodic orbit $x_{s}(t)$ at time $t_{1}$.

A fully equivalent description of the stochastic dynamics (11) is provided by the Fokker-Planck equation 34 for the probability distribution $p(\mathbf{x}, t)$ of an ensemble of particles $\mathbf{x}(t)$,

$$
\frac{\partial}{\partial t} p(\mathbf{x}, t)+\nabla \cdot \mathbf{j}(\mathbf{x}, t)=0
$$

where the probability current density is given by

$$
\mathbf{j}(\mathbf{x}, t):=\mathbf{F}(\mathbf{x}, t) p(\mathbf{x}, t)-\epsilon \mathrm{D} \nabla p(\mathbf{x}, t)
$$

with the positive semidefinite diffusion matrix $\mathrm{D}:=\mathrm{BB}^{T}$.

\section{B. Escape rates}

If we now consider our stochastic dynamics (1) for finite noise-strengths $\epsilon$, it is well-known that a particle $x(t)$ starting in $\mathcal{A}(t)$ will be able to escape out of this basin. To quantify this escape of particles, we first introduce the population $P_{\mathcal{A}}(t)$ in $\mathcal{A}(t)$ :

$$
P_{\mathcal{A}}(t):=\int_{\mathcal{A}(t)} \mathrm{d}^{d} \mathbf{x} p(\mathbf{x}, t)
$$


A natural definition of an "instantaneous rate" $\Gamma(t)$ for such escape events is then given by the relative decrease of this population per unit time 31, 32,

$$
\Gamma(t):=-\dot{P_{\mathcal{A}}}(t) / P_{\mathcal{A}}(t)
$$

We remark that - except for transients at early times - this quantity is independent of the initial conditions at time $t=t_{0}$, provided we start with a distribution that is concentrated within the basin $\mathcal{A}(t)$. We thus make the convenient choice $p\left(\mathbf{x}, t_{0}\right)=\delta\left(\mathbf{x}-\mathbf{x}_{s}\left(t_{0}\right)\right)$. Furthermore, on the time scale of these transients, $\Gamma(t)$ will approach a time-periodic limit, permitting a meaningful definition of the time-averaged rate

$$
\bar{\Gamma}:=\frac{1}{\mathcal{T}} \int_{t}^{t+\mathcal{T}} \mathrm{d} t^{\prime} \Gamma\left(t^{\prime}\right)
$$

In the sequel of the present paper we will deduce analytic expressions for both the instantaneous and the time-averaged rate in the limit of small noise-strength $\epsilon$.

To make progress in this direction, we first note that for weak noise $\epsilon$ the typical time scale of the escape events $1 / \bar{\Gamma}$ is well separated from the time scale of the just mentioned transients [14]. We can thus approximate $P_{\mathcal{A}}(t)$ by its initial value $P_{\mathcal{A}}\left(t_{0}\right)=1$ in the denominator of Eq. (8). Using the Fokker-Planck equation (5) and the divergence theorem, the instantaneous rate reads

$$
\Gamma(t)=\int_{\mathcal{S}(t)} \mathrm{d}^{d-1} \mathbf{n} \cdot[\mathbf{j}(\mathbf{x}, t)-\dot{\mathbf{x}} p(\mathbf{x}, t)] .
$$

Here, the integration is over the entire time-dependent separatrix $\mathcal{S}(t)$ and $\mathbf{n}$ denotes their outer normal vector. Furthermore, the time derivative in $\dot{\mathbf{x}}$ refers to the $t$-dependence of the points on this separatrix, which is determined by the deterministic equation of motion, i.e. Eq. (1) with $\epsilon=0$. Taking this and the definition (6) of the current density into account, one obtains the general result

$$
\Gamma(t)=-\epsilon \int_{\mathcal{S}(t)} \mathrm{d}^{d-1} \mathbf{n} \cdot \mathrm{D} \nabla p(\mathbf{x}, t) .
$$

As expected on naive grounds, a crossing of the separatrix is possible via a diffusive process only.

\section{PATH INTEGRALS AND THE SADDLE-POINT APPROXIMATION}

Let us briefly summarize the path integral description of the dynamics generated by a stochastic differential equation (1). It is well-known from the literature [22, 23, 24, 35, 36, 37, 38, 39, 40, 41, 42, 43, that one can represent the conditional probability density $p\left(\mathbf{x}_{\mathbf{f}}, t_{f} \mid \mathbf{x}_{0}, t_{0}\right)$ to find the particle at time $t_{f}$ at position $\mathbf{x}_{f}$ if it has started at time $t_{0}$ at position $\mathbf{x}_{0}$ as weighted sum over all paths between these two points,

$$
p\left(\mathbf{x}_{f}, t_{f} \mid \mathbf{x}_{0}, t_{0}\right)=\int_{\mathbf{x}\left(t_{0}\right)=\mathbf{x}_{0}}^{\mathbf{x}\left(t_{f}\right)=\mathbf{x}_{f}} \mathcal{D} \mathbf{x}(t) e^{-S[x(t)] / \epsilon} .
$$

Here, $S[x(t)]$ is the effective action or the Onsager-Machlup functional, i.e.

$$
S[x(t)]:=\int_{t_{0}}^{t_{f}} \mathrm{~d} t L(\mathbf{x}(t), \dot{\mathbf{x}}(t), t),
$$

with the corresponding Lagrangian

$$
L(\mathbf{x}, \dot{\mathbf{x}}, t):=\frac{1}{4}[\dot{\mathbf{x}}-\mathbf{F}(\mathbf{x}, t)] \cdot \mathrm{D}^{-1}[\dot{\mathbf{x}}-\mathbf{F}(\mathbf{x}, t)] .
$$

We remark that a prepoint-discretization scheme [41, 42, 43] (not to be confused with the Ito-scheme in stochastic calculus [34]) has been implicitly adopted in the path integral (12) implying the measure $1 / \sqrt{\operatorname{det}(4 \pi \epsilon \Delta t \mathrm{D})}$. Other "discretization schemes" [41, 42, 43] would give rise to a somewhat modified path-integral formalism but would — of course - lead to identical results as far as the actual stochastic dynamics (3) is concerned. 
It should be clear that in general an exact evaluation of the path integral (12) is impossible. Nevertheless, it represents an advantageous starting-point for a systematic weak-noise approximation: For small $\epsilon$, the main contributions to the path integral (12) stem from the surrounding of action minimizing paths. Carrying out a infinite-dimensional saddle-point approximation around these paths, one arrives at

$$
p\left(\mathbf{x}_{f}, t_{f} \mid \mathbf{x}_{0}, t_{0}\right)=\sum_{k} \frac{e^{-\phi_{k}\left(\mathbf{x}_{f}, t_{f}\right) / \epsilon}}{\left[\operatorname{det}\left(4 \pi \epsilon \mathbf{Q}_{k}^{*}\left(t_{f}\right)\right)\right]^{\frac{1}{2}}}[1+\mathcal{O}(\epsilon)]
$$

where the sum runs over all paths $\mathbf{x}_{k}^{*}(t)$ that (locally) minimize the action (13). These obey the Euler-Lagrange equations

$$
\ddot{\mathbf{x}}_{k}^{*}(t)+\left[\mathrm{D}\left(\boldsymbol{\nabla} \mathbf{F}\left(\mathbf{x}_{k}^{*}(t), t\right)\right)^{T} \mathrm{D}^{-1}-\boldsymbol{\nabla} \mathbf{F}\left(\mathbf{x}_{k}^{*}(t), t\right)\right] \dot{\mathbf{x}}_{k}^{*}(t)-\mathrm{D}\left(\boldsymbol{\nabla} \mathbf{F}\left(\mathbf{x}_{k}^{*}(t), t\right)\right)^{T} \mathrm{D}^{-1} \mathbf{F}\left(\mathbf{x}_{k}^{*}(t), t\right)-\frac{\partial}{\partial t} \mathbf{F}\left(\mathbf{x}_{k}^{*}(t), t\right)=0
$$

together with the boundary conditions

$$
\mathbf{x}_{k}^{*}\left(t_{0}\right)=\mathbf{x}_{0} \quad, \quad \mathbf{x}_{k}^{*}\left(t_{f}\right)=\mathbf{x}_{f}
$$

Here and in the following, $\boldsymbol{\nabla} \mathbf{F}(\mathbf{x}, t)$ denotes the Jacobian matrix with components $(\boldsymbol{\nabla} \mathbf{F}(\mathbf{x}, t))_{i j}:=\partial F_{i}(\mathbf{x}, t) / \partial x_{j}$ and the transpose of a matrix $\mathrm{M}$ is written as $\mathrm{M}^{T}$. Introducing the canonical momentum

$$
\mathbf{p}:=\frac{\partial L}{\partial \dot{\mathbf{x}}}=\frac{1}{2} \mathrm{D}^{-1}[\dot{\mathbf{x}}-\mathbf{F}(\mathbf{x}, t)]
$$

we can, via the usual Legendre transformation, pass to the equivalent Hamiltonian dynamics, defined by the Hamiltonian

$$
H(\mathbf{x}, \mathbf{p}, t):=\mathbf{p} \cdot \dot{\mathbf{x}}-L=\mathbf{p} \cdot \mathrm{D} \mathbf{p}+\mathbf{p} \cdot \mathbf{F}(\mathbf{x}, t)
$$

and the corresponding canonical equations

$$
\begin{aligned}
& \dot{\mathbf{x}}_{k}^{*}(t)=\mathbf{F}\left(\mathbf{x}_{k}^{*}(t), t\right)+2 \mathrm{D} \mathbf{p}_{k}^{*}(t) \\
& \dot{\mathbf{p}}_{k}^{*}(t)=-\left(\nabla \mathbf{F}\left(\mathbf{x}_{k}^{*}(t), t\right)\right)^{T} \mathbf{p}_{k}^{*}(t)
\end{aligned}
$$

The value of the action $\phi_{k}\left(\mathbf{x}_{f}, t_{f}\right)$ of a path $\mathbf{x}_{k}^{*}(t)$ is then given by

$$
\phi_{k}\left(\mathbf{x}_{f}, t_{f}\right):=S\left[\mathbf{x}_{k}^{*}\left(t_{f}\right)\right]=\int_{t_{0}}^{t_{f}} \mathrm{~d} t \mathbf{p}_{k}^{*}(t) \cdot \mathrm{D} \mathbf{p}_{k}^{*}(t)
$$

where we have suppressed in favor of notational brevity the dependence on the initial condition $\mathbf{x}_{0}$ at time $t_{0}$. It is noteworthy to point out that in Eq. (19) the momentum enters both quadratically and linearly: the latter linear contribution mimics a magnetic field-like, time-dependent vector potential contribution. For later use, we also recall the well-known result from classical mechanics 44 that the derivative of the extremal action with respect to its endpoint $\mathbf{x}_{f}$ equals the canonical conjugate momentum at time $t_{f}$,

$$
\mathbf{p}_{k}^{*}\left(t_{f}\right)=\frac{\partial \phi_{k}\left(\mathbf{x}_{f}, t_{f}\right)}{\partial \mathbf{x}_{f}} .
$$

The yet unspecified prefactor term $\mathrm{Q}_{k}^{*}\left(t_{f}\right)$ in (15) is given by the solution at time $t_{f}$ of the following second order differential equation

$$
\begin{aligned}
\ddot{\mathrm{Q}}_{k}^{*}(t)-\nabla \mathbf{F}\left(\mathbf{x}_{k}^{*}(t), t\right) & \dot{\mathrm{Q}}_{k}^{*}(t)-\dot{\mathrm{Q}}_{k}^{*}(t) \mathrm{Q}_{k}^{*}(t)^{-1} \mathrm{D}\left(\boldsymbol{\nabla} \mathbf{F}\left(\mathbf{x}_{k}^{*}(t), t\right)\right)^{T} \mathrm{D}^{-1} \mathrm{Q}_{k}^{*}(t) \\
+ & \left\{\boldsymbol{\nabla} \mathbf{F}\left(\mathbf{x}_{k}^{*}(t), t\right) \mathrm{D}\left(\boldsymbol{\nabla} \mathbf{F}\left(\mathbf{x}_{k}^{*}(t), t\right)\right)^{T} \mathrm{D}^{-1}-\mathrm{D}\left(\boldsymbol{\nabla} \mathbf{F}\left(\mathbf{x}_{k}^{*}(t), t\right)\right)^{T} \mathrm{D}^{-1} \nabla \mathbf{F}\left(\mathbf{x}_{k}^{*}(t), t\right)\right. \\
& \left.-\frac{\mathrm{d}}{\mathrm{d} t}\left[\boldsymbol{\nabla} \mathbf{F}\left(\mathbf{x}_{k}^{*}(t), t\right)+\mathrm{D}\left(\boldsymbol{\nabla} \mathbf{F}\left(\mathbf{x}_{k}^{*}(t), t\right)\right)^{T} \mathrm{D}^{-1}\right]+2\left(\mathrm{D} \mathbf{p}_{k}^{*}(t)\right) \cdot\left(\boldsymbol{\nabla} \boldsymbol{\nabla} \mathbf{F}\left(\mathbf{x}_{k}^{*}(t), t\right)\right)\right\} \mathrm{Q}_{k}^{*}(t)=0
\end{aligned}
$$

with initial conditions

$$
\mathrm{Q}_{k}^{*}\left(t_{0}\right)=0, \quad \dot{\mathrm{Q}}_{k}^{*}\left(t_{0}\right)=\mathrm{D}
$$


Here, we have introduced the tensor of third order $(\boldsymbol{\nabla} \nabla \mathbf{F}(\mathbf{x}, t))_{i j l}:=\partial^{2} F_{i}(\mathbf{x}, t) / \partial x_{j} \partial x_{l}$, whereby the scalar product with a matrix appearing in Eq. (23) is defined as

$$
\left[\left(\mathbf{D} \mathbf{p}_{k}^{*}(t)\right) \cdot\left(\boldsymbol{\nabla} \nabla \mathbf{F}\left(\mathbf{x}_{k}^{*}(t), t\right)\right)\right]_{i j}=\sum_{l}\left[\mathbf{D} \mathbf{p}_{k}^{*}(t)\right]_{l} \frac{\left.\partial F_{l}\left(\mathbf{x}_{\mathbf{k}}^{*}, t\right)\right)}{\partial x_{i} \partial x_{j}}
$$

The derivation of Eqs. (23,24) in the framework of the time-discretized version of the path integral (12) proceeds along the same general lines as in the one-dimensional case presented in Ref. [32]. However, the rather technical details are beyond the scope of the present paper.

Though in principle, the prefactor $\mathrm{Q}_{k}^{*}\left(t_{f}\right)$ is completely determined by Eqs. (23,24), it will turn out to be more convenient to consider the following quantity:

$$
\mathrm{G}_{k}^{*}(t):=\frac{1}{2}\left[\mathrm{D}^{-1} \dot{\mathbf{Q}}_{k}^{*}(t) \mathrm{Q}_{k}^{*}(t)^{-1}-\left(\boldsymbol{\nabla} \mathbf{F}\left(\mathbf{x}_{k}^{*}(t), t\right)\right)^{T} \mathrm{D}^{-1}-\mathrm{D}^{-1} \boldsymbol{\nabla} \mathbf{F}\left(\mathbf{x}_{k}^{*}(t), t\right)\right] .
$$

With the help of Eq. (23) one can then verify that $\mathrm{G}_{k}^{*}(t)$ fulfills for $t>t_{0}$ the matrix Riccati equation

$$
\begin{aligned}
\dot{\mathrm{G}}_{k}^{*}(t)= & -2 \mathrm{G}_{k}^{*}(t) \mathrm{D} \mathrm{G}_{k}^{*}(t)-\left(\boldsymbol{\nabla} \mathbf{F}\left(\mathbf{x}_{k}^{*}(t), t\right)\right)^{T} \mathrm{G}_{k}^{*}(t) \\
& -\mathrm{G}_{k}^{*}(t) \boldsymbol{\nabla} \mathbf{F}\left(\mathbf{x}_{k}^{*}(t), t\right)-\mathbf{p}_{k}^{*}(t) \cdot \boldsymbol{\nabla} \boldsymbol{\nabla} \mathbf{F}\left(\mathbf{x}_{k}^{*}(t), t\right) .
\end{aligned}
$$

However, as can be inferred from Eqs. (24, 26), there is no well defined initial condition for $\mathrm{G}_{k}^{*}(t)$ at time $t_{0}$. Instead, we have to content ourselves with the relations

$$
\lim _{t \rightarrow t_{0}}\left[\mathrm{G}_{k}^{*}(t) \mathrm{Q}_{k}^{*}(t)\right]=\frac{1}{2} 1 \text { and } \mathrm{G}_{k}^{*}\left(t_{0}\right)^{-1}=0 .
$$

By tracing over both sides of Eq. (26) one obtains a linear first order differential equation for the determinant det $Q_{k}^{*}(t)$ from Eq. (15):

$$
\frac{\mathrm{d}}{\mathrm{d} t} \operatorname{det} \mathrm{Q}_{k}^{*}(t)=2 \operatorname{Tr}\left[\left(\boldsymbol{\nabla} \mathbf{F}\left(\mathbf{x}_{k}^{*}(t), t\right)\right)^{T}+\mathbf{D} \mathbf{G}_{k}^{*}(t)\right] \operatorname{det} \mathbf{Q}_{k}^{*}(t) .
$$

The initial condition $\operatorname{det} \mathrm{Q}_{k}^{*}\left(t_{0}\right)=0$ follows immediately from Eq. (24).

We conclude this section with two remarks on the above mentioned two different approaches for the calculation of the prefactor in Eq. (15). First, we want to point out that one can identify $\mathrm{G}_{k}^{*}\left(t_{f}\right)$ with the Hessian of the action function $\phi_{k}\left(\mathbf{x}_{f}, t_{f}\right)$ :

$$
\mathrm{G}_{k}^{*}\left(t_{f}\right)=\frac{\partial^{2} \phi_{k}\left(\mathbf{x}_{f}, t_{f}\right)}{\partial \mathbf{x}_{f} \partial \mathbf{x}_{f}}
$$

The proof of this relation proceeds analogously to that presented in Ref. [32] for the one-dimensional case, though the calculational details are by far more involved. Thus, Eq. (27) is equivalent to the matrix Riccati equation for the second derivatives of the action used elsewhere in the literature [24. However, while there this equation is derived by inserting a WKB-type ansatz in the Fokker-Planck equation (5), we entirely work here within the path integral formalism. In particular, we avoid problems commonly encountered due to the non-analytic nature of the WKB-action near the separatrix [22, 24. As a second remark, we note that an advantage of Eqs. (27, 29) over Eq. (23) lies in the fact that in the former set of equations no $\mathrm{D}^{-1}$ terms appear. Since later on we will be interested in the case of singular $\mathrm{D}$, this fact presents a favorable feature from a technical point of view.

\section{EVALUATION OF THE ESCAPE RATE FOR WEAK NOISE}

For small noise strengths $\epsilon$, we can now insert the saddle-point approximation (15) into the rate expression (11). Using Eq. (22), this yields

$$
\Gamma\left(t_{f}\right)=\int_{\mathcal{S}\left(t_{f}\right)} \mathrm{d}^{d-1} \mathbf{n} \cdot \mathrm{D} \sum_{k} \mathbf{p}_{k}^{*}\left(t_{f}\right) \frac{e^{-\phi_{k}\left(\mathbf{x}_{k}^{*}\left(t_{f}\right), t_{f}\right) / \epsilon}}{\left[\operatorname{det}\left(4 \pi \epsilon \mathrm{Q}_{k}^{*}\left(t_{f}\right)\right)\right]^{\frac{1}{2}}}[1+\mathcal{O}(\epsilon)] .
$$

Note that for the integrand the boundary condition (17) with $\mathbf{x}_{f}$ being the integration variable on the separatrix $\mathcal{S}\left(t_{f}\right)$ is implicitly understood. Parametrizing the surface integral (31) by the $d-1$ dimensional vector $\mathbf{s}=\left(s_{1}, \ldots, s_{d-1}\right)^{T}$, 
i.e. $\mathcal{S}\left(t_{f}\right)=:\left\{\mathbf{x}_{\mathrm{sep}}\left(\mathbf{s}, t_{f}\right) \mid \mathbf{s} \in U^{d-1}\right\}$ for a certain subset $U^{d-1} \subseteq \mathbb{R}^{d-1}$ of the $d-1$ dimensional parameter space, we obtain

$$
\Gamma\left(t_{f}\right)=\int \mathrm{d}^{d-1} s \sqrt{g\left(\mathbf{s}, t_{f}\right)} \mathbf{n}\left(\mathbf{s}, t_{f}\right) \cdot \mathrm{D} \sum_{k} \mathbf{p}_{k}^{*}\left(t_{f}\right) \frac{e^{-\phi_{k}\left(\mathbf{x}_{k}^{*}\left(t_{f}\right), t_{f}\right) / \epsilon}}{\left[\operatorname{det}\left(4 \pi \epsilon \mathrm{Q}_{k}^{*}\left(t_{f}\right)\right)\right]^{\frac{1}{2}}}[1+\mathcal{O}(\epsilon)]
$$

where we have introduced the measure

$$
g\left(\mathbf{s}, t_{f}\right):=\operatorname{det}\left(\frac{\partial \mathbf{x}_{\mathrm{sep}}\left(\mathbf{s}, t_{f}\right)}{\partial s_{\nu}} \cdot \frac{\partial \mathbf{x}_{\mathrm{sep}}\left(\mathbf{s}, t_{f}\right)}{\partial s_{\nu^{\prime}}}\right)_{\nu, \nu^{\prime}=1, \ldots d-1}
$$

For weak noise, the main contribution to the integral (32) comes again from the surrounding of the minima of the actions $\phi_{k}\left(\mathbf{x}_{k}^{*}\left(t_{f}\right), t_{f}\right)$, and we can evaluate the integral in a saddle-point approximation. The corresponding extremal condition which determines the values $\mathbf{s}_{k}^{*}$ then assumes the form, cf. also Eq. 22,

$$
\frac{\partial \phi_{k}\left(\mathbf{x}_{\mathrm{sep}}\left(\mathbf{s}_{k}^{*}, t_{f}\right), t_{f}\right)}{\partial s_{k, \nu}^{*}}=\mathbf{p}_{k}^{*}\left(t_{f}\right) \cdot \frac{\partial \mathbf{x}_{\mathrm{sep}}\left(\mathbf{s}_{k}^{*}, t_{f}\right)}{\partial s_{k, \nu}^{*}}=0 \quad(\nu=1, \ldots, d-1)
$$

Consequently, the momentum $\mathbf{p}_{k}^{*}\left(t_{f}\right)$ has to be perpendicular to the separatrix $\mathcal{S}\left(t_{f}\right)$ at the end point $\mathbf{x}_{\mathrm{sep}}\left(\mathbf{s}_{k}^{*}, t_{f}\right)$. Furthermore, we need the Hessian of the action as a function of the parameter vector s. Via Eqs. (22) and (26) it can be expressed as

$$
\begin{aligned}
\frac{\partial^{2} \phi_{k}\left(\mathbf{x}_{\mathrm{sep}}\left(\mathbf{s}_{k}^{*}, t_{f}\right), t_{f}\right)}{\partial s_{k, \nu}^{*} \partial s_{k, \nu^{\prime}}^{*}}= & \frac{\partial \mathbf{x}_{\mathrm{sep}}\left(\mathbf{s}_{k}^{*}, t_{f}\right)}{\partial s_{k, \nu}^{*}} \cdot \mathbf{G}_{k}^{*}\left(t_{f}\right) \frac{\partial \mathbf{x}_{\mathrm{sep}}\left(\mathbf{s}_{k}^{*}, t_{f}\right)}{\partial s_{k, \nu^{\prime}}^{*}} \\
& +\mathbf{p}_{k}^{*}\left(t_{f}\right) \cdot \frac{\partial^{2} \mathbf{x}_{\mathrm{sep}}\left(\mathbf{s}_{k}^{*}, t_{f}\right)}{\partial s_{k, \nu}^{*} \partial s_{k, \nu^{\prime}}^{*}} \quad\left(\nu, \nu^{\prime}=1, \ldots, d-1\right) .
\end{aligned}
$$

Later on, we shall evaluate the last equation within a linearization around the unstable periodic orbit of our deterministic dynamics. In that case, the second term on the r.h.s of Eq. (35) vanishes. The determinant of the Hessian is thus given by the first term, which can be written as (cf. Appendix ):

$$
\operatorname{det}\left(\frac{\partial \mathbf{x}_{\mathrm{sep}}\left(\mathbf{s}_{k}^{*}, t_{f}\right)}{\partial s_{k, \nu}^{*}} \cdot \mathrm{G}_{k}^{*}\left(t_{f}\right) \frac{\partial \mathbf{x}_{\mathrm{sep}}\left(\mathbf{s}_{k}^{*}, t_{f}\right)}{\partial s_{k, \nu^{\prime}}^{*}}\right)_{\nu, \nu^{\prime}=1, \ldots, d-1}=g\left(\mathbf{s}_{k}^{*}, t_{f}\right) \mathbf{n}\left(\mathbf{s}_{k}^{*}, t_{f}\right) \cdot \mathrm{G}_{k}^{*}\left(t_{f}\right)^{-1} \mathbf{n}\left(\mathbf{s}_{k}^{*}, t_{f}\right) \operatorname{det} \mathrm{G}_{k}^{*}\left(t_{f}\right)
$$

Using the fact that the momentum $\mathbf{p}_{k}^{*}\left(t_{f}\right)$ is parallel to the normal vector $\mathbf{n}\left(\mathbf{s}_{k}^{*}, t_{f}\right)$ (cf. Eq. (34)), we then obtain for the rate (32) the important intermediate result

$$
\Gamma\left(t_{f}\right)=\frac{1}{\sqrt{2^{d+1} \pi \epsilon}} \sum_{k} \frac{\mathbf{p}_{k}^{*}\left(t_{f}\right) \cdot \mathrm{D} \mathbf{p}_{k}^{*}\left(t_{f}\right) e^{-\phi_{k}\left(\mathbf{x}_{\mathrm{sep}}\left(\mathbf{s}_{k}^{*}, t_{f}\right), t_{f}\right) / \epsilon}}{\sqrt{\mathbf{p}_{k}^{*}\left(t_{f}\right) \cdot \mathrm{G}_{k}^{*}\left(t_{f}\right)^{-1} \mathbf{p}_{k}^{*}\left(t_{f}\right) \operatorname{det} \mathrm{Q}_{k}^{*}\left(t_{f}\right) \operatorname{det} \mathrm{G}_{k}^{*}\left(t_{f}\right)}}[1+\mathcal{O}(\epsilon)]
$$

\section{A. The action minimizing paths}

To proceed further in the evaluation of the rate formula (37), it is necessary to gain more insight about the nature of the action minimizing paths $\mathbf{x}_{k}^{*}(t)$, whose dynamics is governed by the Hamiltonian equations (20) supplemented by the boundary conditions (17). In view of our choice for the initial conditions (cf. discussion after Eq. (\$)) and our result (37), the latter assume the form

$$
\mathbf{x}_{k}^{*}\left(t_{0}\right)=\mathbf{x}_{s}\left(t_{0}\right) \quad, \quad \mathbf{x}_{k}^{*}\left(t_{f}\right)=\mathbf{x}_{\mathrm{sep}}\left(\mathbf{s}_{k}^{*}, t_{f}\right)
$$

where the parameter values $\mathbf{s}_{k}^{*}$ are restricted by the relations (34).

For given values of $t_{0}$ and $t_{f}$, in the generic case only one of the solutions $\mathbf{x}_{k}^{*}(t)$ of this boundary problem will represent a global minimum of the action (21). Denoting this path for the moment by $\mathbf{x}_{\bar{k}}^{*}(t)$, it is clear that for large time differences $t_{f}-t_{0}$ owing to the form of the action (13) and (14) this path $\mathbf{x}_{\bar{k}}^{*}(t)$ will spent most of its time near a deterministic trajectory, i.e., $\dot{\mathbf{x}}_{\bar{k}}^{*}(t) \approx \mathbf{F}\left(\mathbf{x}_{\bar{k}}^{*}(t), t\right)$. With regard to the boundary conditions 38 , this means that after its start at time $t_{0}$ the path will closely follow the stable periodic orbit $\mathbf{x}_{s}(t)$ for some time. Subsequently it switches over into the vicinity of the separatrix $\mathcal{S}(t)$, thereby accumulating the main part of its action, to remain there until its end at time $t_{f}$. With respect to the position of the end point on the separatrix, if we assume that ultimately all 
deterministic trajectories on the separatrix converge to the unstable periodic orbit $\mathbf{x}_{u}(t)$, the same will also hold true for the path $\mathbf{x}_{\bar{k}}^{*}(t)$, which will thus end nearer and nearer to $\mathbf{x}_{u}(t)$ the more time it is able to spend in a close vicinity of the separatrix. Since the duration of the sojourns near the periodic orbits is long compared to that of the transition in between, the path $\mathbf{x}_{\bar{k}}^{*}(t)$ is often called an "instanton" in the literature.

If we consider now the limiting case $t_{0} \rightarrow-\infty$ and $t_{f} \rightarrow \infty$ (in the following abbreviated as $t_{f}-t_{0} \rightarrow \infty$ ), there exists a well defined limit of $\mathbf{x}_{\bar{k}}^{*}(t)$, in the sense that this path follows ever closer the periodic orbits, while retaining the shape of the intermediate segment. At the same time, its action $S\left[\mathbf{x}_{\bar{k}}^{*}(t)\right]$ converges from above to a finite value. We observe that in the limit $t_{f}-t_{0} \rightarrow \infty$ owing to the time-periodicity of the force field $\mathbf{F}(\mathbf{x}, t)$ the action $S\left[\mathbf{x}_{\bar{k}}^{*}(t+n \mathcal{T})\right]$ is the same for all integers $n$. In other words, the global minimum of the action becomes countable infinitely degenerate. However, one can still safely assume that these minima are well separated in the space of all the paths appearing in (12), provided that the driving is neither too weak, too slow nor too fast. These limiting cases are thus not covered by our theory. We remind the reader of the situation in the static case, where the global minimum is also degenerate. However, while the degeneracy there is continuous in time (Goldstone mode), we are dealing here with a discrete degeneracy.

As a consequence of the fact that the minimizing paths $\mathbf{x}_{k}^{*}(t)$ remain well separated, our rate-formula (37) becomes asymptotically exact for any (arbitrary but fixed) finite values of the driving amplitude and period as the noise strength $\epsilon$ tends to zero. Apart from this fact that in the limit $\epsilon \rightarrow 0$ the $\mathcal{O}(\epsilon)$ correction in the saddle point approximation (15) and thus in (37) vanishes, a more detailed quantitative statement seems difficult. On the other hand, for a given (small) noise strength $\epsilon$, we have to exclude extremely small driving amplitudes and extremely long or short driving periods since this would lead us effectively back to the static (undriven) escape problem, which requires a completely different treatment (especially of the (quasi-) Goldstone mode [27, 38, 39, 45, 46, 47]) than in (15). Put differently, in any of these three asymptotic regimes, the error $\mathcal{O}(\epsilon)$ from (15).37) becomes very large.

For the following, we introduce the symbol $\mathbf{x}_{\mathrm{opt}}^{*}(t)$ for the limit of the path $\mathbf{x}_{\bar{k}}^{*}(t)$ for $t_{f}-t_{0} \rightarrow \infty$, always keeping in mind that this path is only defined modulo time shifts by integer multiples of the driving period $\mathcal{T}$. The corresponding action is defined analogously by

$$
\phi_{\mathrm{opt}}:=S\left[\mathrm{x}_{\mathrm{opt}}^{*}(t)\right] .
$$

Furthermore, all other quantities related to $\mathbf{x}_{\mathrm{opt}}^{*}(t)$ inherit the subscript "opt", for instance $\mathbf{p}_{\text {opt }}^{*}(t)$ to name only one.

Coming back to the case of finite times $t_{0}$ and $t_{f}$, we expect that as precursors of the limit $t_{f}-t_{0} \rightarrow \infty$ there exist besides the global minimum $\mathbf{x}_{\bar{k}}^{*}(t)$ additional, relative minima $\mathbf{x}_{k}^{*}(t)$ with an only slightly larger action. After a suitable relabeling, each of them closely resembles an appropriately shifted "master path" $\mathbf{x}_{\text {opt }}^{*}(t+k \mathcal{T})$ (see Fig. 3). For finite $t_{f}-t_{0}$, we have a finite number of the order $t_{f}-t_{0} / \mathcal{T}$ of such paths. Thus, again without restriction of generality, we can assume that the sum in the rate formula (37) runs from 0 to a maximal value $K\left(t_{f}, t_{0}\right)$ :

$$
0 \leq k \leq K\left(t_{f}, t_{0}\right)=\mathcal{O}\left(\left(t_{f}-t_{0}\right) / \mathcal{T}\right) .
$$

Especially, the path $\mathbf{x}_{0}^{*}(t)$ is the one that stays as long as possible in the vicinity of the stable periodic orbit $\mathbf{x}_{s}(t)$ and starts with its transition towards the separatrix at the latest possible moment.

\section{B. Linearization scheme around periodic orbits}

The discussion in the last section has shown that the paths $\mathbf{x}_{k}^{*}(t)$ spend most of their time near the periodic orbits $\mathbf{x}_{s, u}(t)$ of the deterministic dynamics. Thus, we may gain further insight in their behavior if we linearize the time-dependent force field around these orbits:

$$
\mathbf{F}(\mathbf{x}, t) \approx \mathbf{F}\left(\mathbf{x}_{s, u}(t), t\right)+\nabla \mathbf{F}\left(\mathbf{x}_{s, u}(t), t\right)\left(\mathbf{x}-\mathbf{x}_{s, u}(t)\right) .
$$

Within this approximation, the Hamiltonian equations (20) assume the form

$$
\begin{aligned}
\Delta \dot{\mathbf{x}}_{k}^{*}(t) & =\nabla \mathbf{F}\left(\mathbf{x}_{s, u}(t), t\right) \Delta \mathbf{x}_{k}^{*}(t)+2 \mathrm{D} \mathbf{p}_{k}^{*}(t) \\
\dot{\mathbf{p}}_{k}^{*}(t) & =-\left(\boldsymbol{\nabla} \mathbf{F}\left(\mathbf{x}_{s, u}(t), t\right)\right)^{T} \mathbf{p}_{k}^{*}(t),
\end{aligned}
$$

where we have introduced the deviations from the periodic orbits,

$$
\Delta \mathbf{x}_{k}^{*}(t):=\mathbf{x}_{k}^{*}(t)-\mathbf{x}_{s, u}(t) .
$$

As we shall see later, for the evaluation of the rate formula (37), one only needs the solution for the momentum equation in the second line of Eq. (42). According to Floquet's theory [48, this solution can be obtained from the 


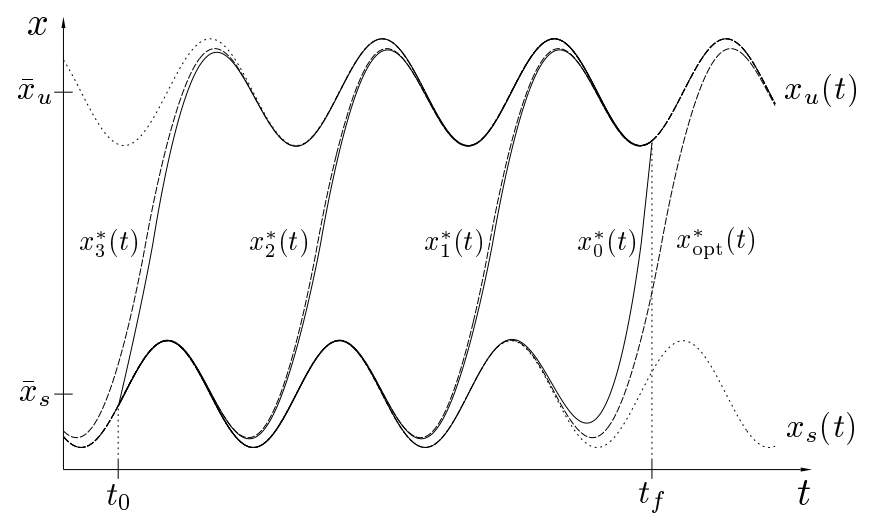

FIG. 3: Action minimizing paths $x_{k}^{*}(t), k=0, \ldots, K\left(t_{f}, t_{0}\right)=3$ (solid) and corresponding master paths $x_{\mathrm{opt}}^{*}(t+k \mathcal{T})($ dashed) between stable and unstable periodic orbits (dotted). Depicted is the one-dimensional, overdamped case $d=1$ with an additively, harmonically driven piecewise parabolic potential from Ref. [31, 32].

eigenvalues and the left eigenvectors of the eigenvalue problem

$$
\left[\frac{\mathrm{d}}{\mathrm{d} t}+\nabla \mathbf{F}\left(\mathbf{x}_{s, u}(t), t\right)\right] \boldsymbol{\Phi}_{s, u}^{\alpha}(t)=\lambda_{s, u}^{\alpha} \boldsymbol{\Phi}_{s, u}^{\alpha}(t), \mathbf{\Phi}_{s, u}^{\alpha}(t+\mathcal{T})=\boldsymbol{\Phi}_{s, u}^{\alpha}(t), \alpha=1, \ldots, d
$$

together with the corresponding right eigenvectors $\boldsymbol{\Phi}_{s, u}^{\dagger, \alpha}(t)$, i.e. the solutions of Eq. 44$)$ with $\left(\boldsymbol{\nabla} \mathbf{F}\left(\mathbf{x}_{s, u}(t), t\right)\right)^{T}$. Upon proper normalization of these eigenvectors at equal times, i.e.

$$
\boldsymbol{\Phi}_{s, u}^{\dagger, \alpha}(t) \cdot \boldsymbol{\Phi}_{s, u}^{\beta}(t)=\delta_{\alpha \beta}
$$

we are able to write the solution for the momentum equation as

$$
\mathbf{p}_{k}^{*}(t)=\sum_{\alpha} e^{-\lambda_{s, u}^{\alpha}\left(t-t_{1}\right)} \mathbf{\Phi}_{s, u}^{\alpha}\left(t_{1}\right) \cdot \mathbf{p}_{k}^{*}\left(t_{1}\right) \boldsymbol{\Phi}_{s, u}^{\dagger, \alpha}(t),
$$

where $t_{1}$ are arbitrary reference times for which the linearization (41) is valid. Since for the master path we have $\Delta \mathbf{x}_{\mathrm{opt}}^{*}(t) \rightarrow 0$ for $t \rightarrow \pm \infty$, it follows with Eq. (42) that $\lim _{t \rightarrow \pm \infty} \mathbf{p}_{\mathrm{opt}}^{*}(t)=0$. Consequently, we must require $\operatorname{Re} \lambda_{u}^{\alpha}>0\left(\operatorname{Re} \lambda_{s}^{\alpha}<0\right)$ for all $\alpha$ with $\boldsymbol{\Phi}_{u, s}^{\alpha}\left(t_{1}\right) \cdot \mathbf{p}_{\text {opt }}^{*}\left(t_{1}\right) \neq 0$. In the following, we denote sums over this subset of eigenvectors by an apostrophe on the sum sign.

With respect to the prefactor quantities $\mathrm{G}_{k}^{*}(t)$ and $\operatorname{det} \mathrm{Q}_{k}^{*}(t)$, we first observe that within the linearization (41) the matrix Riccati equation (27) assumes the form

$$
\dot{\mathrm{G}}_{k}^{*}(t)=-2 \mathrm{G}_{k}^{*}(t) D \mathrm{G}_{k}^{*}(t)-\left(\boldsymbol{\nabla} \mathbf{F}\left(\mathbf{x}_{s, u}(t), t\right)\right)^{T} \mathrm{G}_{k}^{*}(t)-\mathrm{G}_{k}^{*}(t) \boldsymbol{\nabla} \mathbf{F}\left(\mathbf{x}_{s, u}(t), t\right) .
$$

A transformation to the inverse matrix $\mathrm{G}_{k}^{*}(t)^{-1}$ yields the linear matrix differential equation

$$
\frac{\mathrm{d}}{\mathrm{d} t}\left[\mathrm{G}_{k}^{*}(t)^{-1}\right]=2 \mathrm{D}+\mathrm{G}_{k}^{*}(t)^{-1}\left(\boldsymbol{\nabla} \mathbf{F}\left(\mathbf{x}_{s, u}(t), t\right)\right)^{T}+\boldsymbol{\nabla} \mathbf{F}\left(\mathbf{x}_{s, u}(t), t\right) \mathrm{G}_{k}^{*}(t)^{-1},
$$

where the initial condition is given by Eq. (28) as

$$
\mathrm{G}_{k}^{*}\left(t_{0}\right)^{-1}=0 .
$$

Furthermore, multiplication of Eq. (47) with $\mathrm{G}_{k}^{*}(t)^{-1}$ shows that

$$
\operatorname{Tr}\left[\dot{\mathrm{G}}_{k}^{*}(t) \mathrm{G}_{k}^{*}(t)^{-1}\right]=-2 \operatorname{Tr}\left[\left(\boldsymbol{\nabla} \mathbf{F}\left(\mathbf{x}_{s, u}(t), t\right)\right)^{T}+\mathbf{D ~ G}_{k}^{*}(t)\right] .
$$

With the help of the linearized version of Eq. (29) we then find for the last two terms of the product appearing in the denominator of our rate expression (37) the result

$$
\operatorname{det} \mathrm{G}_{k}^{*}(t) \operatorname{det} \mathrm{Q}_{k}^{*}(t)=\text { const. }=: \mu_{s, u} .
$$


Note that while $\mu_{s}$ is fixed for all paths $\mathbf{x}_{k}^{*}(t)$ by the initial condition $(28)$ to the value $\mu_{s}=2^{-d}, \mu_{u}$ depends both on the index $k$ and on the explicit form of the time-dependent force field $\mathbf{F}(\mathbf{x}, t)$. Later on, we only need $\mu_{u}$ for the master path, which we denote by

$$
\mu_{\mathrm{opt}}:=\lim _{t \rightarrow \infty} \operatorname{det} \mathrm{G}_{\mathrm{opt}}^{*}(t) \operatorname{det} \mathrm{Q}_{\mathrm{opt}}^{*}(t) .
$$

The rest of the product in the denominator of (37) can be rewritten by using the equation of motion (48) for $\mathrm{G}_{k}^{*}(t)^{-1}$ together with the Hamiltonian equation (42) for $\mathbf{p}_{k}^{*}(t)$. This yields

$$
\frac{\mathrm{d}}{\mathrm{d} t}\left[\mathbf{p}_{k}^{*}(t) \cdot \mathrm{G}_{k}^{*}(t)^{-1} \mathbf{p}_{k}^{*}(t)\right]=2 \mathbf{p}_{k}^{*}(t) \cdot \mathbf{D} \mathbf{p}_{k}^{*}(t)
$$

or equivalently in integrated form

$$
\mathbf{p}_{k}^{*}(t) \cdot \mathrm{G}_{k}^{*}(t)^{-1} \mathbf{p}_{k}^{*}(t)=\mathbf{p}_{k}^{*}\left(t_{1}\right) \cdot \mathrm{G}_{k}^{*}\left(t_{1}\right)^{-1} \mathbf{p}_{k}^{*}\left(t_{1}\right)+2 \int_{t_{1}}^{t} \mathrm{~d} t^{\prime} \mathbf{p}_{k}^{*}\left(t^{\prime}\right) \cdot \mathrm{D} \mathbf{p}_{k}^{*}\left(t^{\prime}\right)
$$

For the master path $\mathbf{x}_{\mathrm{opt}}^{*}(t)$, the integral on the right hand side of the last equation has to converge in the limit $t \rightarrow \infty$ in order that the action $\phi_{\text {opt }}$ assumes a finite value. Hence, the quantity on the left hand side is also well defined in this limit and we obtain for $t \rightarrow \infty$ after a subsequent renaming $t_{u} \rightarrow t$

$$
\mathbf{p}_{\mathrm{opt}}^{*}(t) \cdot \mathrm{G}_{\mathrm{opt}}^{*}(t)^{-1} \mathbf{p}_{\mathrm{opt}}^{*}(t)=q_{\mathrm{opt}}-\mathbf{p}_{\mathrm{opt}}^{*}(t) \cdot \mathrm{A}_{u}(t) \mathbf{p}_{\mathrm{opt}}^{*}(t)
$$

Here we have exploited Eq. (46) for the master path and furthermore introduced the abbreviations

$$
\begin{aligned}
q_{\mathrm{opt}} & :=\lim _{t \rightarrow \infty} \mathbf{p}_{\mathrm{opt}}^{*}(t) \cdot \mathrm{G}_{\mathrm{opt}}^{*}(t)^{-1} \mathbf{p}_{\mathrm{opt}}^{*}(t), \\
\mathrm{A}_{u}(t) & :=2 \sum_{\alpha \beta}^{\prime} \boldsymbol{\Phi}_{u}^{\alpha}(t) \boldsymbol{\Phi}_{u}^{\beta}(t)^{T} \int_{t}^{\infty} \mathrm{d} t^{\prime} e^{-\left(\lambda_{u}^{\alpha}+\lambda_{u}^{\beta}\right)\left(t^{\prime}-t\right)} \boldsymbol{\Phi}_{u}^{\dagger, \alpha}\left(t^{\prime}\right) \cdot \mathbf{D} \boldsymbol{\Phi}_{u}^{\dagger, \beta}\left(t^{\prime}\right) .
\end{aligned}
$$

Note that owing to the time-periodicity of the Floquet solutions, $\mathrm{A}_{u}(t)$ is also a periodic function of $t$ :

$$
\mathrm{A}_{u}(t+k \mathcal{T})=\mathrm{A}_{u}(t) \text { for all times } t .
$$

\section{Approximation in terms of the master path}

After having gained sufficient insight into the nature of the paths $\mathbf{x}_{k}^{*}(t)$, we now come back to the evaluation of the rate expression (37). The main idea is to approximate all quantities related to the paths $\mathbf{x}_{k}^{*}(t)$ in terms of the corresponding master path, since, as discussed in Sect. IVA, both resemble each other closely. However, while the boundary conditions for the master path are $\mathbf{x}_{\mathrm{opt}}^{*}(t)-\mathbf{x}_{s}(t) \rightarrow 0$ for $t \rightarrow-\infty$ and $\mathbf{x}_{\mathrm{opt}}^{*}(t)-\mathbf{x}_{u}(t) \rightarrow 0$ for $t \rightarrow \infty$, the paths $\mathbf{x}_{k}^{*}(t)$ have to satisfy Eq. (38). From the results of our previous work [32], we know that while we can safely neglect these deviations at the initial time $t_{0}$, the boundary at time $t_{f}$ has to be treated more carefully. First, we modify the upper boundary condition for $\mathbf{x}_{\mathrm{opt}}^{*}(t)$ by requiring that

$$
t_{k}:=t_{f}+k \mathcal{T}, \quad \mathbf{x}_{k}:=\mathbf{x}_{\mathrm{opt}}^{*}\left(t_{k}\right)
$$

are the new end time and end point, respectively. In other words, we simply truncate the path $\mathbf{x}_{\text {opt }}^{*}(t+k \mathcal{T})$ corresponding to $\mathbf{x}_{k}^{*}(t)$ at the time $t_{f}$. Obviously, this new path still satisfies the Hamiltonian equations (20) and is thus an extremizing path. The value of its action follows from the definitions (21) and (39) as

$$
\phi_{\mathrm{opt}}\left(\mathbf{x}_{k}, t_{k}\right):=\int_{-\infty}^{t_{k}} \mathrm{~d} t \mathbf{p}_{\mathrm{opt}}^{*}(t) \cdot \mathbf{D} \mathbf{p}_{\mathrm{opt}}^{*}(t)=\phi_{\mathrm{opt}}-\int_{t_{k}}^{\infty} \mathrm{d} t \mathbf{p}_{\mathrm{opt}}^{*}(t) \cdot \mathbf{D} \mathbf{p}_{\mathrm{opt}}^{*}(t) .
$$

Next, we express the action (21) of the path $\mathbf{x}_{k}^{*}(t)$ by expanding the one belonging to the associated master path $\mathbf{x}_{\mathrm{opt}}^{*}(t+k \mathcal{T})$ in powers of the difference $\delta \mathbf{x}_{k}^{*}\left(t_{f}\right):=\mathbf{x}_{k}^{*}\left(t_{f}\right)-\mathbf{x}_{\mathrm{opt}}^{*}\left(t_{k}\right)=\Delta \mathbf{x}_{k}^{*}\left(t_{f}\right)-\Delta \mathbf{x}_{\mathrm{opt}}^{*}\left(t_{k}\right)$ between their endpoints,

$$
\phi_{k}\left(\mathbf{x}_{\mathrm{sep}}\left(\mathbf{s}_{k}^{*}, t_{f}\right), t_{f}\right)=\phi_{\mathrm{opt}}\left(\mathbf{x}_{k}, t_{k}\right)+\frac{\partial \phi_{\mathrm{opt}}\left(\mathbf{x}_{k}, t_{k}\right)}{\partial \mathbf{x}_{k}} \cdot \delta \mathbf{x}_{k}^{*}\left(t_{f}\right)+\cdots .
$$


The second term on the right hand side can be rewritten as $\mathbf{p}_{\text {opt }}^{*}\left(t_{k}\right) \cdot \Delta \mathbf{x}_{k}^{*}\left(t_{f}\right)-\mathbf{p}_{\mathrm{opt}}^{*}\left(t_{k}\right) \cdot \Delta \mathbf{x}_{\mathrm{opt}}^{*}\left(t_{k}\right)$. Here, the first scalar product is zero, as can been seen as follows: We know that the endpoint $\mathbf{x}_{k}^{*}\left(t_{f}\right)$ lies one the separatrix $\mathcal{S}\left(t_{f}\right)$ and thus a deterministic solution $\mathbf{x}_{\text {det }}(t)$ starting at time $t_{k}$ from $\mathbf{x}_{\text {det }}\left(t_{k}\right)=\mathbf{x}_{k}^{*}\left(t_{f}\right)$ will for all times stay on $\mathcal{S}(t)$. Its deviation from the unstable periodic orbit is denoted by $\Delta \mathbf{x}_{\text {det }}(t)$. Within our linearization (41) one can then readily verify with the help of the dynamical equation (42) that $\mathrm{d}\left[\mathbf{p}_{\mathrm{opt}}^{*}(t) \cdot \Delta \mathbf{x}_{\mathrm{det}}(t)\right] / \mathrm{d} t=0$. Hence, this scalar product is a constant of motion and we can infer that

$$
\mathbf{p}_{\mathrm{opt}}^{*}\left(t_{k}\right) \cdot \Delta \mathbf{x}_{k}^{*}\left(t_{f}\right)=\mathbf{p}_{\mathrm{opt}}^{*}\left(t_{k}\right) \cdot \Delta \mathbf{x}_{\mathrm{det}}\left(t_{k}\right)=\lim _{t \rightarrow \infty}\left[\mathbf{p}_{\mathrm{opt}}^{*}(t) \cdot \Delta \mathbf{x}_{\mathrm{det}}(t)\right]=0 .
$$

Here, the last equality follows from the boundedness of $\mathbf{x}_{\text {det }}(t)$ near the saddle point (cf. the discussion below Eq. (2)) together with $\mathbf{p}_{\mathrm{opt}}^{*}(t) \rightarrow 0$ for $t \rightarrow \infty$.

The second scalar product, $\mathbf{p}_{\mathrm{opt}}^{*}\left(t_{k}\right) \cdot \Delta \mathbf{x}_{\mathrm{opt}}^{*}\left(t_{k}\right)$, can be determined by a similar consideration: The linearized Hamiltonian dynamics (42) yields

$$
\frac{\mathrm{d}}{\mathrm{d} t}\left[\mathbf{p}_{\mathrm{opt}}^{*}(t) \cdot \Delta \mathbf{x}_{\mathrm{opt}}^{*}(t)\right]=2 \mathbf{p}_{\mathrm{opt}}^{*}(t) \cdot \mathrm{D} \mathbf{p}_{\mathrm{opt}}^{*}(t)
$$

and since again this scalar product has to vanish in the limit $t \rightarrow \infty$, we obtain

$$
\mathbf{p}_{\mathrm{opt}}^{*}\left(t_{k}\right) \cdot \Delta \mathbf{x}_{\mathrm{opt}}^{*}\left(t_{k}\right)=-2 \int_{t_{k}}^{\infty} \mathrm{d} t \mathbf{p}_{\mathrm{opt}}^{*}(t) \cdot \mathrm{D} \mathbf{p}_{\mathrm{opt}}^{*}(t) .
$$

Altogether, the approximation (61) for the value of the action of the path $\mathbf{x}_{k}^{*}(t)$ thus takes the form

$$
\phi_{k}\left(\mathbf{x}_{\mathrm{sep}}\left(\mathbf{s}_{k}^{*}, t_{f}\right), t_{f}\right)=\phi_{\mathrm{opt}}+\int_{t_{f}}^{\infty} \mathrm{d} t \mathbf{p}_{\mathrm{opt}}^{*}(t+k \mathcal{T}) \cdot \mathrm{D} \mathbf{p}_{\mathrm{opt}}^{*}(t+k \mathcal{T})+\cdots
$$

The $k$-dependence of the exponential term in the rate expression (37) is thus reduced to a simple sum of exponential factors as given by the time-dependence (46) of the integrand in (65). With respect to the prefactor terms in the rate expression (37), we already now from the results of our previous work 32 that up to corrections of the order $\mathcal{O}\left(\left|\mathbf{p}_{\text {opt }}^{*}\left(t_{k}\right)\right|^{2}\right)$ we can directly substitute all quantities belonging to the path $\mathbf{x}_{k}^{*}(t)$ by those of the corresponding master path $\mathbf{x}_{\mathrm{opt}}^{*}(t+k \mathcal{T})$. Consistently, we also neglect the terms on the right hand side of Eq. (55), which are of the same order.

\section{Rate formula}

After the discussion in the previous section, it is just a matter of collecting everything in order to arrive at the central result of the present work, namely the asymptotic value of the instantaneous escape rate,

$$
\Gamma(t) \simeq \sqrt{\epsilon} \alpha_{\mathrm{opt}} e^{-\phi_{\mathrm{opt}} / \epsilon} \kappa_{\mathrm{opt}}(t, \epsilon)
$$

Here, we have introduced the quantities

$$
\begin{aligned}
\alpha_{\mathrm{opt}} & :=\left[2^{d+1} \pi \mathcal{T}^{2} \lim _{t \rightarrow \infty} \mathbf{p}_{\mathrm{opt}}^{*}(t) \cdot \mathrm{G}_{\mathrm{opt}}^{*}(t)^{-1} \mathbf{p}_{\mathrm{opt}}^{*}(t) \operatorname{det} \mathrm{Q}_{\mathrm{opt}}^{*}(t) \operatorname{det} \mathrm{G}_{\mathrm{opt}}^{*}(t)\right]^{-\frac{1}{2}}, \\
\kappa_{\mathrm{opt}}(t, \epsilon) & :=\mathcal{T} \sum_{k=0}^{K\left(t, t_{0}\right)} \frac{\mathbf{p}_{\mathrm{opt}}^{*}(t+k \mathcal{T}) \cdot \mathrm{D} \mathbf{p}_{\mathrm{opt}}^{*}(t+k \mathcal{T})}{\epsilon} \exp \left[-\frac{1}{\epsilon} \int_{t}^{\infty} \mathrm{d} t^{\prime} \mathbf{p}_{\mathrm{opt}}^{*}\left(t^{\prime}+k \mathcal{T}\right) \cdot \mathrm{D} \mathbf{p}_{\mathrm{opt}}^{*}\left(t^{\prime}+k \mathcal{T}\right)\right] .
\end{aligned}
$$

With the help of Eqs. (46, 57) and the time-periodicity of the Floquet solutions $\boldsymbol{\Phi}_{u}^{\alpha}(t)$ we can rewrite the last expression as

$$
\begin{aligned}
\kappa_{\mathrm{opt}}(t, \epsilon) & =\mathcal{T} \sum_{k=0}^{K\left(t, t_{0}\right)} \frac{\mathbf{b}_{\mathrm{opt}}(t) \cdot \mathrm{B}_{k}(t)^{T} \mathrm{D} \mathrm{B}_{k}(t) \mathbf{b}_{\mathrm{opt}}(t)}{\epsilon} \exp \left\{-\frac{\mathbf{b}_{\mathrm{opt}}(t) \cdot \mathrm{B}_{k}(t)^{T} \mathrm{~A}_{u}(t) \mathrm{B}_{k}(t) \mathbf{b}_{\mathrm{opt}}(t)}{2 \epsilon}\right\}, \\
\mathrm{B}_{k}(t) & :=\sum_{\alpha}^{\prime} e^{-\lambda_{u}^{\alpha} k \mathcal{T}} \boldsymbol{\Phi}_{u}^{\dagger, \alpha}(t) \boldsymbol{\Phi}_{u}^{\alpha}(t)^{T}=\mathrm{B}_{k}(t+\mathcal{T}) \\
\mathbf{b}_{\mathrm{opt}}(t) & :=\lim _{\hat{t} \rightarrow \infty} \sum_{\alpha}^{\prime} e^{-\lambda_{u}^{\alpha}(t-\hat{t})} \boldsymbol{\Phi}_{u}^{\alpha}(\hat{t}) \cdot \mathbf{p}_{\mathrm{opt}}^{*}(\hat{t}) \boldsymbol{\Phi}_{u}^{\dagger, \alpha}(t) .
\end{aligned}
$$


Since $\mathrm{B}_{k}(t)$ in $(70)$ is a sum of terms that exponentially decrease with $k$, there is a competition in the sum (69) between a pre-exponential factor which quickly decreases with $k$ and an exponential term increasing with $k$. The main contribution to this sum thus comes from a few $k$ values around a number $\hat{k}(t)$ that is for small noise strength $\epsilon$ much larger than 0 but at the same moment, for large enough $t_{f}-t_{0}$, still much smaller than $K\left(t_{f}, t_{0}\right)$. Hence, up to an exponentially small error in $\epsilon$, we can extend the summation range in Eq. (69) and thus in Eq. (68) to all integers $k$. This allows us to identify two important features of the time-dependence of $\kappa_{\text {opt }}(t, \epsilon)$. To prove the first one, the time-periodicity

$$
\kappa_{\mathrm{opt}}(t+\mathcal{T}, \epsilon)=\kappa_{\mathrm{opt}}(t, \epsilon)
$$

we merely have to shift the summation index in Eq. (68) by 1. For asymptotic times and small noise strengths, the instantaneous escape rate $(66)$ is thus, as expected, a periodic function of the time $t$. To establish the second property, we use furthermore that the pre-exponential term in Eq. (68) is just the negative time-derivative of the expression in the exponential. Again up to an exponentially small correction, we then obtain

$$
\frac{1}{\mathcal{T}} \int_{t}^{t+\mathcal{T}} \mathrm{d} t^{\prime} \kappa_{\text {opt }}\left(t^{\prime}, \epsilon\right)=1 .
$$

Inserting the asymptotic rate expression (66) into the definition (9) for the time-averaged rate and using the last identity, we thus arrive at the second main result of the present work, namely

$$
\bar{\Gamma} \simeq \sqrt{\epsilon} \alpha_{\mathrm{opt}} e^{-\phi_{\mathrm{opt}} / \epsilon}
$$

Hence, the noise-strength dependence of the time-averaged rate is of the form of an Arrhenius-type, exponentially leading term times an $\epsilon$-dependent prefactor. Note that for a system in thermal equilibrium i.e. for a time-independent force field $\mathbf{F}(\mathbf{x})$, the escape rate is given by an exponentially leading Arrhenius factor, which contains the barrier against the escape, multiplied by an $\epsilon$-independent prefactor, which depends only on local properties of the force field at the barrier and in the well [19]. In comparison with this equilibrium rate structure, we observe two crucial differences in (74). First, in the present, non-equilibrium situation, both the effective potential barrier in the exponential and the prefactor depend in a non-trivial way on global properties of the force field $\mathbf{F}(\mathbf{x}, t)$, and can thus in general only be determined by means of a numerical computation. Second, the non-equilibrium case exhibits an $\epsilon$-dependence of the prefactor.

\section{EXPLICIT RESULTS FOR A METASTABLE PIECEWISE PARABOLIC POTENTIAL}

While, in general, one has to resort to numerical methods for the evaluation of the rate expression (66), in the special case of a particle moving in a one-dimensional piecewise parabolic potential with an additive sinusoidal driving (3) a complete analytical treatment is possible. In the following, we work out the simplest such example with two parabolic pieces, and compare the so obtained analytical predictions with numerical results from a Monte-Carlo simulation of the stochastic dynamics (3).

Let us thus consider the force field deriving from the piecewise parabolic potential of the form

$$
\begin{aligned}
& V(x \leq 0)=\frac{1}{2} m \omega_{s}^{2}\left[\left(x-\bar{x}_{s}\right)^{2}-\bar{x}_{s}^{2}\right] \\
& V(x \geq 0)=\frac{1}{2} m \omega_{u}^{2}\left[\left(x-\bar{x}_{u}\right)^{2}-\bar{x}_{u}^{2}\right],
\end{aligned}
$$

where $\bar{x}_{s}<0$ and $\bar{x}_{u}>0$ denote the position of the potential minimum and maximum, respectively, with corresponding curvatures

$$
m \omega_{s}^{2}>0 \text { and } m \omega_{u}^{2}<0
$$

Note that within our notation $\omega_{u}$ is an imaginary number. To ensure the continuity of the corresponding force field $F(x, t)$,

$$
\begin{aligned}
& F(x \leq 0, t)=-m \omega_{s}^{2}\left(x-\bar{x}_{s}\right)+A \sin (\Omega t) \\
& F(x \geq 0, t)=-m \omega_{u}^{2}\left(x-\bar{x}_{u}\right)+A \sin (\Omega t),
\end{aligned}
$$


at the point $x=0$, we have to impose the additional restriction $\omega_{s}^{2} \bar{x}_{s}=\omega_{u}^{2} \bar{x}_{u}$. Selecting as independent model parameters $\omega_{s}^{2}, \omega_{u}^{2}$, and the static potential barrier $\Delta V:=V\left(\bar{x}_{u}\right)-V\left(\bar{x}_{s}\right)$, the fixed points $x_{s, u}$ can be expressed through

$$
\omega_{s}^{2} \bar{x}_{s}=\omega_{u}^{2} \bar{x}_{u}=-\sqrt{\frac{2 \Delta V}{m} \frac{\omega_{s}^{2}\left|\omega_{u}\right|^{2}}{\omega_{s}^{2}+\left|\omega_{u}\right|^{2}}}
$$

If we require furthermore that the periodic orbits $x_{s, u}(t)$ do not cross the point $x=0$, i.e.

$$
x_{s}(t)<0<x_{u}(t)
$$

for all times $t$, which is granted if and only if the conditions

$$
A^{2}<m^{2}\left[\gamma^{2} \Omega^{2}+\left(\Omega^{2}-\omega_{s, u}^{2}\right)^{2}\right] \bar{x}_{s, u}^{2}
$$

are fulfilled for both the "s" and the " $\mathrm{u}$ " indices, we obtain

$$
x_{s, u}(t)=\bar{x}_{s, u}-\frac{A}{m} \frac{\gamma \Omega \cos (\Omega t)+\left(\Omega^{2}-\omega_{s, u}^{2}\right) \sin (\Omega t)}{\gamma^{2} \Omega^{2}+\left(\Omega^{2}-\omega_{s, u}^{2}\right)^{2}} .
$$

For the determination of the master path $x_{\mathrm{opt}}^{*}(t)$, we use the specific form (4) of the force field and of the diffusion matrix to express the Hamiltonian equations of motion (20) in the form of the second order differential equations

$$
\begin{aligned}
\Delta \ddot{x}_{\mathrm{opt}}^{*}(t)+\gamma \Delta \dot{x}_{\mathrm{opt}}^{*}(t)+\omega_{s, u}^{2} \Delta x_{\mathrm{opt}}^{*}(t) & =2 \frac{\gamma}{m} p_{v, \mathrm{opt}}^{*}(t) \\
\ddot{p}_{v, \mathrm{opt}}^{*}(t)-\gamma \dot{p}_{v, \mathrm{opt}}^{*}(t)+\omega_{s, u}^{2} p_{v, \mathrm{opt}}^{*}(t) & =0 .
\end{aligned}
$$

Here, the index "s" ("u") applies for all times $t$ where $x_{\mathrm{opt}}^{*}(t) \leq 0\left(x_{\mathrm{opt}}^{*}(t) \geq 0\right)$. Furthermore, we have already set the strength $\delta$ of the auxiliary noise term appearing in (化 to 0, since Eq. (82) and all the following relations remain well-defined for a singular diffusion matrix D. The system (82) of linear equations can be readily solved, if we restrict ourselves to the case where the master path $x_{\text {opt }}^{*}(t)$ crosses the point $x=0$ exactly once, say at time $t_{1}$,

$$
x_{\mathrm{opt}}^{*}(t)=0 \Longleftrightarrow t=t_{1} \text {. }
$$

Notice that owing to the form of the Hamiltonian equations (32) both $x_{\mathrm{opt}}^{*}(t)$ and $p_{v \text {,opt }}^{*}(t)$ have to be continuously differentiable at this time $t_{1}$. For the following considerations it is convenient to introduce the frequencies

$$
\lambda_{s, u}^{ \pm}=-\frac{\gamma}{2} \pm \sqrt{\frac{\gamma^{2}}{4}-\omega_{s, u}^{2}}
$$

which are the Floquet eigenvalues from Eq. (44). We observe that $\lambda_{u}^{-}<0$, such that the respective terms do not appear in the sums (57,70,71). The corresponding Floquet states are given by

$$
\boldsymbol{\Phi}_{s, u}^{ \pm}(t)=\frac{1}{\sqrt{\lambda_{s, u}^{+}-\lambda_{s, u}^{-}}}\left(\begin{array}{c}
1 \\
\lambda_{s, u}^{ \pm}
\end{array}\right) \quad \text { and } \quad \boldsymbol{\Phi}_{s, u}^{\dagger, \pm}(t)=\frac{1}{\sqrt{\lambda_{s, u}^{+}-\lambda_{s, u}^{-}}}\left(\begin{array}{c}
\lambda_{s, u}^{ \pm}+\gamma \\
1
\end{array}\right) \text {. }
$$

Hence, the matrices $\mathrm{B}_{k}(t)$ from Eq. (70) take the form

$$
\mathrm{B}_{k}(t)=\frac{e^{-\lambda_{u}^{+} k \mathcal{T}}}{\lambda_{u}^{+}-\lambda_{u}^{-}}\left(\begin{array}{cc}
\lambda_{u}^{+}+\gamma & 1 \\
\lambda_{u}^{+}\left(\lambda_{u}^{+}+\gamma\right) & \lambda_{u}^{+}
\end{array}\right)
$$

Taking into account the boundary conditions $\Delta x_{\text {opt }}^{*}(t) \rightarrow 0$ and $p_{v \text {,opt }}^{*}(t) \rightarrow 0$ for $t \rightarrow \pm \infty$, we obtain for the solutions of Eq. 82)

$$
\begin{aligned}
& \Delta x_{\mathrm{opt}}^{*}\left(t \leq t_{1}\right)=\frac{1}{\lambda_{s}^{+}-\lambda_{s}^{-}}\left\{\left[\frac{1}{m} p_{v, \mathrm{opt}}^{*}\left(t_{1}\right)-\lambda_{s}^{+} x_{s}\left(t_{1}\right)\right] e^{-\lambda_{s}^{-} \cdot\left(t-t_{1}\right)}-\left[\frac{1}{m} p_{v, \mathrm{opt}}^{*}\left(t_{1}\right)-\lambda_{s}^{-} x_{s}\left(t_{1}\right)\right] e^{-\lambda_{s}^{+} \cdot\left(t-t_{1}\right)}\right\} \\
& \Delta x_{\mathrm{opt}}^{*}\left(t \geq t_{1}\right)=\frac{1}{\lambda_{u}^{+}}\left\{\left[\frac{1}{m} p_{v, \mathrm{opt}}^{*}\left(t_{1}\right)-\lambda_{u}^{+} x_{u}\left(t_{1}\right)\right] e^{\lambda_{u}^{-} \cdot\left(t-t_{1}\right)}-\frac{1}{m} p_{v, \mathrm{opt}}^{*}\left(t_{1}\right) e^{-\lambda_{u}^{+} \cdot\left(t-t_{1}\right)}\right\} \\
& p_{v, \mathrm{opt}}^{*}\left(t \leq t_{1}\right)=\frac{1}{\lambda_{s}^{+}-\lambda_{s}^{-}}\left\{\left[-\lambda_{s}^{-} p_{v, \mathrm{opt}}^{*}\left(t_{1}\right)+m \omega_{s}^{2} x_{s}\left(t_{1}\right)\right] e^{-\lambda_{s}^{-} \cdot\left(t-t_{1}\right)}+\left[\lambda_{s}^{+} p_{v, \mathrm{opt}}^{*}\left(t_{1}\right)-m \omega_{s}^{2} x_{s}\left(t_{1}\right)\right] e^{-\lambda_{s}^{+} \cdot\left(t-t_{1}\right)}\right\} \\
& p_{v, \mathrm{opt}}^{*}\left(t \geq t_{1}\right)=p_{v, \mathrm{opt}}^{*}\left(t_{1}\right) e^{-\lambda_{u}^{+} \cdot\left(t-t_{1}\right)} .
\end{aligned}
$$


Next one can infer from the above mentioned requirement of a continuous first derivative at time $t$ of both $x_{\text {opt }}^{*}(t)$ and $p_{v, \text { opt }}^{*}(t)$ the two relations

$$
\begin{aligned}
& p_{v, \mathrm{opt}}^{*}\left(t_{1}\right)=m \lambda_{u}^{+}\left[x_{u}\left(t_{1}\right)+\frac{\dot{x}_{s}\left(t_{1}\right)-\dot{x}_{u}\left(t_{1}\right)}{\lambda_{u}^{-}}\right] \\
& p_{v, \mathrm{opt}}^{*}\left(t_{1}\right)=m \frac{\omega_{s}^{2}}{\omega_{u}^{2}} \lambda_{u}^{+} x_{s}\left(t_{1}\right) .
\end{aligned}
$$

These relations can be used to fix the two so far unknown quantities $t_{1}$ and $p_{v, \text { opt }}^{*}\left(t_{1}\right)$ in the above expressions: $\mathrm{A}$ straightforward, but somewhat tedious calculation yields

$$
\begin{aligned}
& \tan \left(\Omega t_{1}\right)=\frac{1}{\Omega} \frac{\left(\omega_{s}^{2}-\Omega^{2}\right) \lambda_{u}^{+}+\gamma \Omega^{2}}{\omega_{s}^{2}-\Omega^{2}-\gamma \lambda_{u}^{+}} \\
& p_{v, \text { opt }}^{*}\left(t_{1}\right)=m \lambda_{u}^{+} \bar{x}_{u}+\omega_{s}^{2} \frac{\lambda_{u}^{+}}{\lambda_{u}^{-}} \frac{A}{\Omega} \frac{\cos \left(\Omega t_{1}\right)}{\omega_{s}^{2}-\Omega^{2}-\gamma \lambda_{u}^{+}} .
\end{aligned}
$$

Obviously, Eq. (89) has two solutions within each driving period $\mathcal{T}$. We anticipate that only one corresponds to a minimum of the action, and hence to the master path. Thus, we fix $t_{1}$ (up to the usual degeneracy under $t \rightarrow t+\mathcal{T}$ ) by requiring additionally that

$$
\frac{A}{\Omega} \frac{\cos \left(\Omega t_{1}\right)}{\omega_{s}^{2}-\Omega^{2}-\gamma \lambda_{u}^{+}}>0
$$

Combining (89-191) we arrive at

$$
p_{v, \mathrm{opt}}^{*}\left(t_{1}\right)=m \lambda_{u}^{+} \bar{x}_{u}+\frac{|A| \omega_{s}^{2}\left|\omega_{u}\right|^{2}}{\lambda_{u}^{-} \nu^{4}}>0
$$

where we have introduced the frequency $\nu$ via

$$
\nu^{4}:=\left[\left(\gamma^{2} \Omega^{2}+\left(\Omega^{2}-\omega_{s}^{2}\right)^{2}\right)\left(\omega_{u}^{4}+\Omega^{2} \lambda_{u}^{-2}\right)\right]^{1 / 2}
$$

To conclude the discussion of the master path, we note that in order to check the consistency of the solution (87) with the condition (83) it is necessary to solve a transcendental equation, which has to be done numerically. Qualitatively, one expects a break-down of Eq. (33) in the deterministically underdamped regime, i.e. $\gamma / 2<\omega_{s}$, where the path $\left(x_{\mathrm{opt}}(t), v_{\mathrm{opt}}(t)\right)$ in phase space leaves the vicinity of the stable periodic orbit by way of a spiraling orbit which crosses the line $x=0$ several times.

Inserting $p_{v, \text { opt }}^{*}(t)$ from Eq. (87) and (88,92) into the definition (21, 39) yields the action of the master path:

$$
\phi_{\mathrm{opt}}=\Delta V\left[1-\left|\frac{A^{2} \omega_{s}^{2} \omega_{u}^{2}\left(\omega_{s}^{2}+\left|\omega_{u}\right|^{2}\right)}{2 m \Delta V \nu^{8}}\right|^{1 / 2}\right]^{2} .
$$

The periodic driving thus leads to an "effective potential barrier" $\phi_{\text {opt }}$ which is smaller than the static barrier $\Delta V$ to which it reduces in the limits $A \rightarrow 0$ or $\Omega \rightarrow \infty$. While $\phi_{\text {opt }}$ is monotonically decreasing with increasing driving amplitude $A$, the dependency on the driving frequency $\Omega$ is more complicated. In particular one observes resonance behavior near the frequency $\omega_{s}$ of the bottom well. Had we chosen the opposite inequality in Eq. (91), a plus instead of the minus sign would have appeared in the Eq. (94). Thus, the condition (91) does indeed single out the minimum of the action and thus the desired solution for the master path $x_{\mathrm{opt}}^{*}(t)$. Using the result for $p_{v, \mathrm{opt}}^{*}\left(t_{1}\right)$ from Eq. 92), we obtain the following explicit expression for $\mathbf{b}_{\mathrm{opt}}(t)$ from Eq. (71):

$$
\mathbf{b}_{\mathrm{opt}}(t)=\left(\begin{array}{c}
\lambda_{u}^{+}+\gamma \\
1
\end{array}\right) p_{v, \mathrm{opt}}^{*}\left(t_{1}\right) e^{-\lambda_{u}^{+}\left(t-t_{1}\right)}
$$

Finally, we turn to the determination of the prefactor quantity $\alpha_{\mathrm{opt}}$. We first note that in the present case the linear differential equation (48) does not only hold approximatively but is exact for all times $t \neq t_{1}$. Owing to $t_{0} \rightarrow-\infty$, the solution has approached its stationary value, and we obtain

$$
\mathrm{G}_{\mathrm{opt}}^{*}\left(t<t_{1}\right) \equiv\left(\begin{array}{cc}
m \omega_{s}^{2} & 0 \\
0 & m
\end{array}\right)
$$


and hence with Eq. (28)

$$
\operatorname{det} \mathrm{Q}_{\mathrm{opt}}^{*}\left(t<t_{1}\right)=\frac{1}{4 m \omega_{s}^{2}}
$$

At this point, we make use of the fact that $\operatorname{det} \mathrm{Q}_{\mathrm{opt}}^{*}(t)$ is continuous at time $t_{1}$ according to Eq. (29), while $\mathrm{G}_{\mathrm{opt}}^{*}(t)$ jumps due to Eq. (27). With $\partial^{2} F(x, t) / \partial x^{2}=m\left(\omega_{s}^{2}-\omega_{u}^{2}\right) \delta(x)$ one thus can infer that

$$
\mathrm{G}_{\mathrm{opt}}^{*}\left(t_{1}+0\right)=\left(\begin{array}{cc}
\left(\omega_{s}^{2}+\left|\omega_{u}\right|^{2}\right) \frac{m \dot{x}_{\mathrm{opt}}^{*}\left(t_{1}\right)-p_{v, \mathrm{opt}}^{*}\left(t_{1}\right)}{\dot{x}_{\mathrm{opt}}^{*}\left(t_{1}\right)}-\left|\omega_{u}\right|^{2} & 0 \\
0 & m
\end{array}\right) .
$$

This yields together with Eq. (51), which again is exact for the piecewise parabolic potential, the intermediate result

$$
\lim _{t \rightarrow \infty} \operatorname{det} \mathrm{G}_{\mathrm{opt}}^{*}(t) \operatorname{det} \mathrm{Q}_{\mathrm{opt}}^{*}(t)=\frac{1}{4 m \omega_{s}^{2}}\left[\left(\omega_{s}^{2}+\left|\omega_{u}\right|^{2}\right) \frac{m \dot{x}_{\mathrm{opt}}^{*}\left(t_{1}\right)-p_{v, \mathrm{opt}}^{*}\left(t_{1}\right)}{\dot{x}_{\mathrm{opt}}^{*}\left(t_{1}\right)}-m\left|\omega_{u}\right|^{2}\right] .
$$

Applying two times Eq. (54), one time for $t=t_{0} \rightarrow-\infty$ using the initial condition (49) and a second time for $t \rightarrow \infty$, yields after some algebra another necessary quantity, namely

$$
\begin{aligned}
& \lim _{t \rightarrow \infty} \mathbf{p}_{\mathrm{opt}}^{*}(t) \cdot \mathrm{G}_{\mathrm{opt}}^{*}(t)^{-1} \mathbf{p}_{\mathrm{opt}}^{*}(t)= \\
& p_{x, \mathrm{opt}}^{*}\left(t_{1}\right)^{2}\left\{\left[\left(\omega_{s}^{2}+\left|\omega_{u}\right|^{2}\right) \frac{m \dot{x}_{\mathrm{opt}}^{*}\left(t_{1}\right)-p_{v, \mathrm{opt}}^{*}\left(t_{1}\right)}{\dot{x}_{\mathrm{opt}}^{*}\left(t_{1}\right)}-m\left|\omega_{u}\right|^{2}\right]^{-1}-\frac{1}{m \omega_{s}^{2}}\right\}+2 \phi_{\mathrm{opt}}
\end{aligned}
$$

Inserting these expressions into the definition (67) of $\alpha_{\text {opt }}$ and using Eqs. (20), (92) and (94) we arrive at

$$
\alpha_{\mathrm{opt}}=\left[4 \pi \mathcal{T}^{2} \frac{m \dot{x}_{\mathrm{opt}}^{*}\left(t_{1}\right)-p_{v, \mathrm{opt}}^{*}\left(t_{1}\right)}{m \dot{x}_{\mathrm{opt}}^{*}\left(t_{1}\right)} \phi_{\mathrm{opt}}\right]^{-1 / 2}
$$

Again, our choice for the sign in Eq. 91] is justified a posteriori, since it guarantees that $m \dot{x}_{\text {opt }}^{*}\left(t_{1}\right)-p_{v, \text { opt }}^{*}\left(t_{1}\right)$ is a positive quantity. Eventually, together with Eqs. (78), (81), (89), (90) and (92), the last expression can be rewritten as

$$
\alpha_{\mathrm{opt}}=\left[\frac{|A|\left[\Omega^{2} \lambda_{u}^{-2}-\omega_{s}^{2}\left|\omega_{u}\right|^{2}\right]+\sqrt{\frac{2 m \Delta V \nu^{8}}{\omega_{s}^{-2}+\left|\omega_{u}\right|^{-2}}}}{16 \pi^{3}|A| \lambda_{u}^{-2} \phi_{\mathrm{opt}}}\right]^{1 / 2} .
$$

We remark that the structure of the results (94) and (102) closely resembles those in the overdamped case, as obtained in Ref. [31, 32]. In particular, this limiting case $m \rightarrow 0$ is correctly reproduced by (94) and (102).

We have checked our above analytical predictions by comparing them with results from Monte-Carlo simulations of the stochastic dynamics (3), which yield the mean time $\overline{t_{\text {exit }}}$ necessary for an exit out of the driven well. The time-averaged rate is related to this quantity via 49]

$$
\bar{\Gamma}=\left(\overline{t_{\text {exit }}}\right)^{-1}
$$

Since the necessary simulation times diverge exponentially with decreasing noise strength $\epsilon$ (cf. (74) and (102)), the numerical determination of the mean exit time becomes impossible for extremely small $\epsilon$. For similar reasons, the numerical determination of the time-resolved rates $\Gamma(t)$ is ruled out.

Figures 4 -6 depict the time-averaged rate $\bar{\Gamma}$ as a function of various system parameters. Parameter regions for which condition (83) is not fulfilled are indicated, which in particular applies for the case of weak damping $\eta$ and the vicinity of the deterministic resonance at $\Omega^{2}=\omega_{s}^{2}-\gamma^{2} / 2$, as discussed above. Outside of these regions, already for a noise strength of $\epsilon=1$, the agreement between theory and simulation is very good (compare also inset of Fig. 6).

Furthermore, as one can see in Fig. 4 , the theory breaks down both for small and large driving frequencies $\Omega$, if the noise strength $\epsilon$ is kept fixed. However, for $\epsilon \rightarrow 0$, we again observe the convergence of numerically determined rate towards the theoretical approximation (cf. inset of Fig. A). This behavior is in full accordance with our predictions from Sect. IV A. 


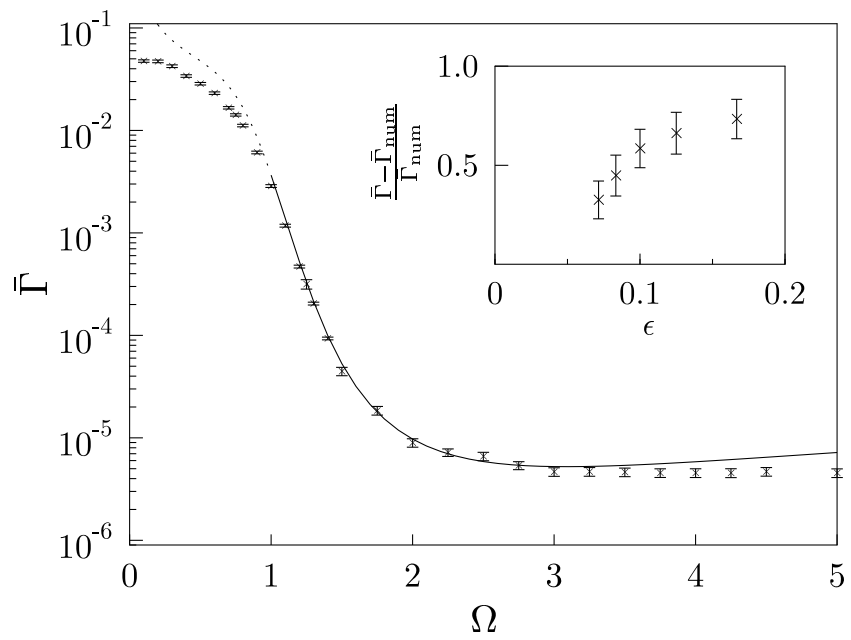

FIG. 4: Time-averaged rate $\bar{\Gamma} v s$. driving frequency $\Omega$ for $\bar{x}_{s}=\omega_{u}^{2}=-1, \bar{x}_{u}=\omega_{s}^{2}=m=\eta=\Delta V=A=1, \epsilon=0.1$ (dimensionless units). Crosses: Inverse mean first exit time from simulations of Eq. (3) (error bars: mean square deviation of mean value). Solid (dotted) line: Analytical prediction (74) (condition (83) not fulfilled). Inset: Convergence behavior of $\bar{\Gamma}$ for $\epsilon \rightarrow 0$ for fixed driving frequency $\Omega=5$ (all other parameters as in main panel).

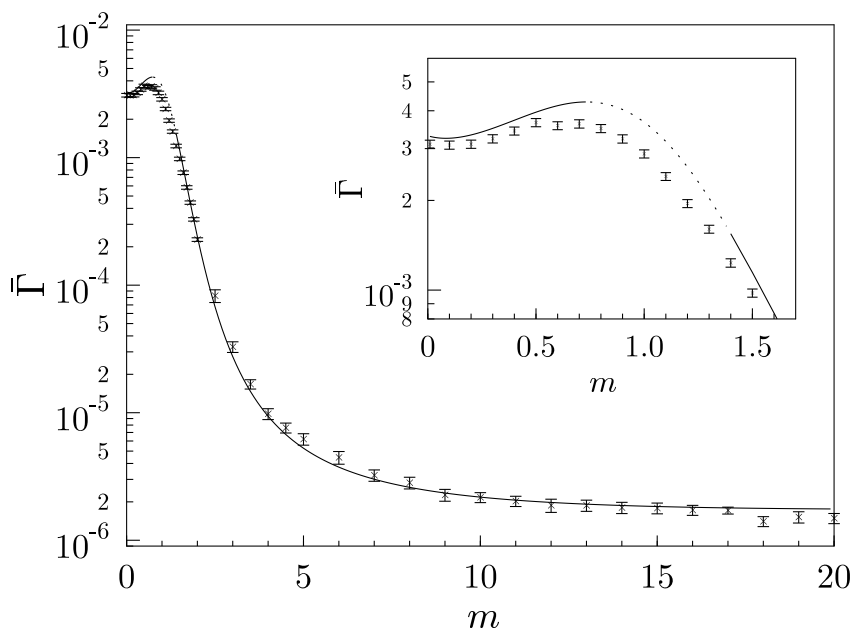

FIG. 5: Time-averaged rate $\bar{\Gamma} v s$. particle mass $m$ for $\bar{x}_{s}=m \omega_{u}^{2}=-1, \bar{x}_{u}=m \omega_{s}^{2}=\eta=\Delta V=A=\Omega=1, \epsilon=0.1$ (dimensionless units). Crosses: Inverse mean first exit time from simulations of Eq. (3) (error bars: mean square deviation of mean value). Solid (dotted) line: Analytical prediction (74) (condition (83) not fulfilled). Inset: Magnification of small $m$ regime. Note that the deterministic resonance lies at $m=\sqrt{1 / 2} \approx 0.707$.

\section{CONCLUSIONS}

With the present work, we have generalized earlier results for the noise-activated escape in time-periodically driven one-dimensional overdamped systems to an arbitrary number of dimensions. The basic idea of the path-integral approach put forward is that it is necessary to sum over all local, nearby minima of the relevant action that contribute to the escape rate. For asymptotically weak noise, these minima are well separated in the space of all paths, and the contribution of each of them can be obtained by a standard saddle-point approximation of the path-integral. This 


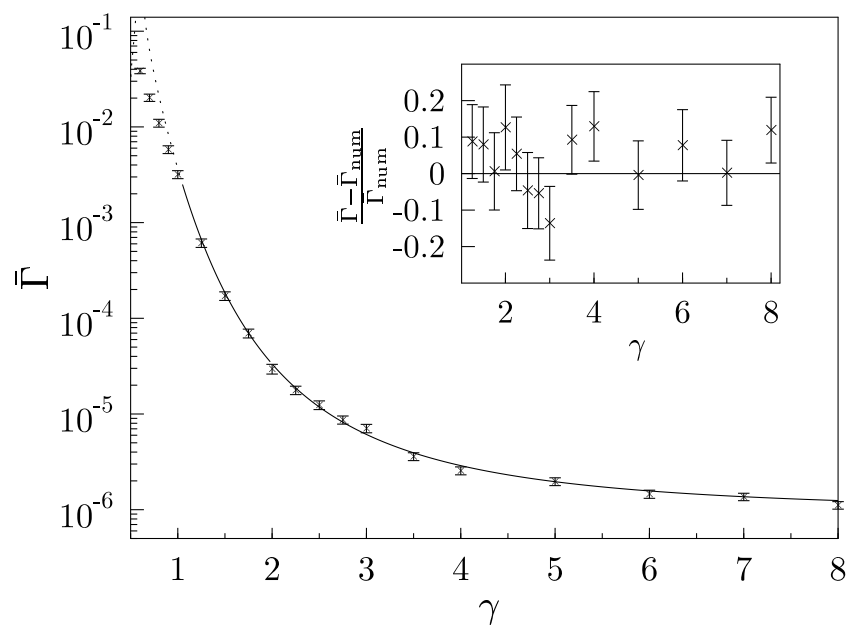

FIG. 6: Time-averaged rate $\bar{\Gamma} v s$. damping coefficient $\gamma=\eta / m$ for $\bar{x}_{s}=\omega_{u}^{2}=-1, \bar{x}_{u}=\omega_{s}^{2}=m=\Delta V=A=\Omega=1, \epsilon=0.1$ (dimensionless units). Crosses: Inverse mean first exit time from simulations of Eq. (3) (error bars: mean square deviation of mean value). Solid (dotted) line: Analytical prediction (74) (condition (83) not fulfilled). Inset: Relative difference between analytical prediction and numerical result as a function of $\gamma$.

behavior is in strong contrast to the undriven situation, where the occurrence of a (quasi-) Goldstone mode requires a more sophisticated treatment of the associated prefactor.

Our central results express the time-instantaneous rate (66) and the time-averaged rate (74) in terms of quantities belonging to a master path, which has in general to be obtained numerically as the solution of a minimization problem. However, the structure of the result already reveals two noteworthy, salient differences compared to the static case: both the exponentially leading Arrhenius factor and the prefactor not only depend on the relative barrier height and the local properties of the potential inside the well and at the barrier region, respectively, but in addition these two quantities depend as well sensitively on the global behavior of the time-dependent metastable potential. Additionally, a $\sqrt{\epsilon}$-dependence of the rate prefactor appears as a consequence of the non-equilibrium situation. Finally, we have been able to derive closed analytical rate expressions for a distinctive case, namely the sinusoidally driven Kramers problem with a metastable potential formed by two parabolas.

We also like to point out here that the treatment of driven escape in meta-stable potential landscapes by use of path integral methods is close in spirit to prominent work by Jozef T. Devreese wherein he pioneered the challenging problem of polarons in magnetic fields [50, 51]: in both cases the effective Hamiltonian involves a vector-potential like contribution which is linear in the canonical momentum variable, see Eq. (19). Then, as pointed out repeatedly by Jozef, the standard variational principle based on the Feynman-Jensen inequality no longer applies [51, 52], but requires instead an appropriate modification $[52$.

\section{Acknowledgments}

This work has been supported by the Studienstiftung des deutschen Volkes (J.L.), the Graduiertenkolleg GRK-283, the Sonderforschungsbereich SFB-486 (J.L.,P.H.), and the Sonderforschungsbereich SFB-613 (P.R.). One of us (P.H.) likes to acknowledge many elucidating and stimulating scientific discussions with Jozef T. Devreese who, as we all know, is still very active not only within his beloved physics of polarons but increasingly also within the timely area of physics on the nanoscale. Jozef is still young enough to appreciate and to contribute great science!

\section{APPENDIX}

In this appendix, we shall prove Eq. (36). Let us first abbreviate the $d \times d-1$ matrix $\mathbf{S}$ composed of the vectors $\partial \mathbf{x}_{\mathrm{sep}}\left(\mathbf{s}_{k}^{*}, t_{f}\right) / \partial s_{k, \nu}^{*}, \nu=1, \ldots, d-1$ as columns. For notational brevity, we suppress here and in the following the asterix, all indices $k$, and the time arguments. Indicating furthermore with $\mathrm{S}^{i}, i=1, \ldots, d$, the $d-1 \times d-1$ matrix, 
which consists of $\mathrm{S}$ without row $i$ and with $\mathrm{G}_{j}^{i}, i, j=1, \ldots, d$, the matrix without row $i$ and column $j$, we can apply a generalized version of the determinant multiplication theorem [53] to the left hand side of Eq. (36]), yielding

$$
\operatorname{det}(\mathrm{SGS})=\sum_{i j} \operatorname{det} \mathrm{S}^{j} \operatorname{det} \mathrm{G}_{j}^{i} \operatorname{det} \mathrm{S}^{i}
$$

Using a standard relation between the cofactors and the inverse of a matrix 53]

$$
(-1)^{i+j} \operatorname{det} \mathrm{G}_{j}^{i}=\left(\mathrm{G}^{-1}\right)_{j i} \operatorname{det} \mathrm{G}
$$

we obtain the intermediate result

$$
\operatorname{det}(\mathrm{S} G \mathrm{~S})=\mathbf{u} \cdot \mathrm{G}^{-1} \mathbf{u} \operatorname{det} \mathrm{G}
$$

Here, we have introduced the $d$-dimensional vector $u_{i}:=(-1)^{i+d} \operatorname{det} S^{i}, i=1, \ldots, d$. A comparison with Eq. (36) shows that the proof is complete, if we are able to demonstrate that $\mathbf{u}=\sqrt{g} \mathbf{n}$. To this end, we define another auxiliary quantity, namely the $d \times d$ matrix $\mathrm{M}:=(\mathrm{S} \mid \mathbf{n})$. Since the normal vector $\mathbf{n}$ of the separatrix $\mathcal{S}$ is orthogonal to all columns of $\mathrm{S}$, the determinant of $\mathrm{M}$ is given by (cf. also Eq. (33))

$$
\operatorname{det} M=\sqrt{\operatorname{det} M^{T} M}=\sqrt{\operatorname{det} S^{T S}}=\sqrt{g}
$$

For the same reason, one has $\left(\mathrm{M}^{-1}\right)_{d i}=\mathrm{M}_{i d}=n_{i}, i=1, \ldots, d$, and thus

$$
u_{i}=(-1)^{i+d} \operatorname{det} \mathrm{M}_{d}^{i}=\operatorname{det} \mathrm{M}\left(\mathrm{M}^{-1}\right)_{d i}=\sqrt{g} n_{i} .
$$

[1] R. de Vivie-Riedle, H. Rabitz, K.-L. Kompa, special issue: Laser control of quantum dynamics, Chem. Phys. 267, Nos. 1-3 (2001).

[2] M. Shapiro, Adv. Chem. Phys. 114, 123 (2000).

[3] E.H. Serpersu and T.Y. Tsong, J. Membr. Biol. 74, 191 (1983); J. Biol. Chem. 259, 7155 (1984); D.S. Liu, R.D. Astumian, and T.Y. Tsong, ibid 265, 7260 (1990).

[4] P. Hänggi and R. Bartussek in Nonlinear Physics of Complex Systems, Lecture Notes in Physics, Vol. 476, 294 -308 (1996); J. Parisi, S.C. Müller, and W. Zimmermann (eds.), (Springer, Berlin, 1996.)

[5] R.D. Astumian, Science 276, 917 (1997).

[6] F. Jülicher, A. Ajdari, and J. Prost, Rev. Mod. Phys. 69, 1269 (1997).

[7] P. Reimann, Phys. Rep. 361, 57 (2002).

[8] P. Reimann and P. Hänggi, Appl. Phys. A 75, 169 (2002).

[9] R.D. Astumian and P. Hänggi, Brownian Motors, Physics Today, 55, No. 11 (november), 33-39 (2002).

[10] P. Jung and P. Hänggi, Phys. Rev. A 44, 8032 (1991).

[11] L. Gammaitoni, P. Hänggi, P. Jung, and F. Marchesoni, Rev. Mod. Phys 70, 223 (1998).

[12] K. Wiesenfeld and F. Moss, Nature 373, 33 (1995).

[13] P. Hänggi, CHEMPHYSCHEM 3, 285 (2002).

[14] P. Hänggi, P. Talkner, and M. Borkovec, Rev. Mod. Phys. 62, 251 (1990).

[15] G.R. Fleming and P. Hänggi (editors), Activated barrier crossing; applications in Physics, Chemistry, and Biology, World Scientific, Singapore 1993.

[16] P. Talkner and P. Hänggi (editors), New trends in Kramers' Reaction Rate Theory, Kluwer, Dordrecht 1995.

[17] H.A. Kramers, Physica (Utrecht) 7, 284 (1940).

[18] R. Landauer and J.A. Swanson, Phys. Rev. 121, 1668 (1961).

[19] J.S. Langer, Ann. Phys. (N.Y.) 54, 258 (1969).

[20] B.J. Matkowsky and Z. Schuss, SIAM J. Appl. Math. 33, 365 (1977).

[21] P. Talkner, Z. Phys. B 68, 201 (1987).

[22] R. Graham and T. Tél, Phys. Rev. A 31, 1109 (1985).

[23] S.J.B. Einchcomb and A.J. McKane, Phys. Rev. E 51, 2974 (1995).

[24] R.S. Maier and D.L. Stein, SIAM J. Appl. Math. 57, 752 (1997).

[25] R. Graham and T. Tél, J. Stat. Phys. 35729 (1984); 37709 (1984).

[26] P. Jung, Phys. Rep. 234, 175 (1993).

[27] V.N. Smelyanskiy, M.I. Dykman, and B. Golding, Phys. Rev. Lett. 82, 3193 (1999).

[28] P. Jung, Z. Phys. B 76, 521 (1989).

[29] P. Talkner, New J. Phys. 1, 4 (1999).

[30] P. Reimann, R. Bartussek, R. Häußler, and P. Hänggi, Phys. Lett. A 215, 26 (1996). 
[31] J. Lehmann, P. Reimann, P. Hänggi, Phys. Rev. Lett. 84, 1639 (2000).

[32] J. Lehmann, P. Reimann, P. Hänggi, Phys. Rev. E. 62, 6282 (2000).

[33] R.S. Maier and D.L. Stein, Phys. Rev. Lett. 86, 3942 (2001).

[34] H. Risken, The Fokker-Planck Equation, Springer, Berlin 1984.

[35] D. Ludwig, SIAM Review 17, 605 (1975).

[36] H.S. Wio, P. Colet, M. San Miguel, L. Pesquera, and M.A. Rodriguez, Phys. Rev. A 40, 7312 (1989).

[37] M.I. Freidlin and A.D. Wentzell, Random Perturbations of Dynamical Systems, Springer, New York 1984.

[38] U. Weiss and W. Häffner, p. 311 in Functional Integration, edited by J.P. Antoine and E. Tirapegui, Plenum, New York 1980 .

[39] B. Caroli, C. Caroli, and B. Roulet, J. Stat. Phys. 26, 83 (1981).

[40] L.S. Schulman, Techniques and Applications of Path Integration, Wiley, New York 1981.

[41] F. Langouche, D. Roekaerts, and E. Tirapegui, Functional Integration and Semiclassical Expansions, Vol. 10 of Mathematics and its Applications, D. Reidel, Dordrecht, Holland, Boston, USA 1982.

[42] P. Hänggi, Z. Physik B 75, 275 (1989).

[43] P. Hänggi, in: Path Integrals from meV to MeV: Tutzing 1992, edited by H. Grabert, A. Inomata, L.S. Schulman, and U. Weiss, (World Scientific, Singapore 1993), pp: 289-301.

[44] H. Goldstein, Classical Mechanics, Addison-Wesley, Reading, Massachusetts, USA 1980.

[45] S. Coleman, in: The Whys of Subnuclear Physics, edited by A. Zichichi, (Plenum, New York, USA 1979), pp: 805-920.

[46] U. Weiss, Phys. Rev. A 25, 2444 (1982).

[47] U. Weiss, H. Grabert, P. Hänggi, and P. Riseborough, Phys. Rev. B 35, 9535 (1987).

[48] G. Floquet, Ann. de l'Ecole Norm. Sup. 12, 47 (1883).

[49] P. Reimann, G.J. Schmid und P. Hänggi, Phy. Rev. E 60, R1 (1999) Rapid Communication.

[50] F.M. Peeters and J.T. Devreese, Phys. Rev. B 31, 3689 (1985); J.T. Devreese, Polarons, in Encyclopedia of Applied Physics 14, 383 (1996).

[51] F. Brosens and J.T. Devreese, Phys. Rev. B 54, 9792 (1996).

[52] J.T. Devreese and F. Brosens, Phys. Rev. B 45, 6459 (1992).

[53] A.C. Aitken, Determinants and Matrices, Oliver and Boyd, Edinburgh (1956). 